\title{
A!
}

This is an electronic reprint of the original article.

This reprint may differ from the original in pagination and typographic detail.

Puska, M. J.; Nieminen, R. M.

\section{Theory of hydrogen and helium impurities in metals}

Published in:

Physical Review B

DOI:

10.1103/PhysRevB.29.5382

Published: 15/05/1984

Document Version

Publisher's PDF, also known as Version of record

Please cite the original version:

Puska, M. J., \& Nieminen, R. M. (1984). Theory of hydrogen and helium impurities in metals. Physical Review $B$, 29(10), 5382-5397. https://doi.org/10.1103/PhysRevB.29.5382

This material is protected by copyright and other intellectual property rights, and duplication or sale of all or part of any of the repository collections is not permitted, except that material may be duplicated by you for your research use or educational purposes in electronic or print form. You must obtain permission for any other use. Electronic or print copies may not be offered, whether for sale or otherwise to anyone who is not an authorised user. 


\title{
Theory of hydrogen and helium impurities in metals
}

\author{
M. J. Puska \\ Laboratory of Physics, Helsinki University of Technology, SF-02150 Espoo, Finland \\ R. M. Nieminen \\ Department of Physics, University of Jyväskylä, SF-40100 Jyväskylä, Finland
}

(Received 10 November 1983)

\begin{abstract}
A powerful computational scheme is presented for calculating the static properties of light interstitials in metallic hosts. The method entails (i) the construction of the potential-energy field using the quasiatom concept, (ii) the wave-mechanical solution of the impurity distribution ("zero-point motion"), (iii) calculation of the forces exerted on the adjacent host atoms and their displacements, and (iv) iteration to self-consistency. We investigate self-trapping phenomena in bcc and fcc metals in detail, and calculate both the ground and low-lying excited states. Implications of the wavemechanical or band picture to diffusion mechanisms and inelastic scattering experiments are discussed. Impurities treated are $\mu^{+}, \mathrm{H}, \mathrm{D}, \mathrm{T}$, and $\mathrm{He}$, and particular attention is paid to isotope effects among the hydrogenic impurities. It is argued that especially for $\mu^{+}$and $\mathrm{H}$ the quantum nature of the impurity is crucial. The calculated results are in agreement with a wealth of experimental data.
\end{abstract}

\section{INTRODUCTION}

The behavior of light impurities (notably helium, the hydrogen isotopes, and positive muons) remains a subject of considerable interest in metal physics. ${ }^{1}$ It is a complicated subject for two reasons. Firstly, the impurity provides a large perturbation for the host-metal electrons. This leads to a strong electronic interaction which may be basically repulsive in nature, as for inert gases, or a more involved "hybridization" or "bonding-antibonding" interaction for chemically active species such as hydrogen. Secondly, the impurity couples strongly to the host-ion coordinates, which leads to polaron-type lattice distortions. The wave mechanics of the degrees of freedom of the light-mass impurity is important in this context. Both of these aspects must enter into any satisfactory description of impurity-related phenomena: ground-state electronic structure, energetics and site assignment, lattice displacements in the self-trapped state, local-mode excitations, diffusion-related effects, etc.

The treatment of the electronic structure of impurities in solids has shown remarkable recent progress. Techniques for self-consistent $a b$ initio calculations ${ }^{2}$ have been developed with increasing computational power and accuracy. However, even within the local-density approximation for exchange and correlation, such calculations are still time consuming and costly, and only feasible mainly for relatively simple, high-symmetry situations. However, the progress in this area has made it possible to create simpler schemes more easily adaptable to complicated low-symmetry situations. A particularly interesting approach is the effective-medium or quasiatom theory proposed independently by Stott and Zaremba ${ }^{3}$ and Nørskov and Lang. ${ }^{4}$ In it one formulates the electronic interaction between the impurity and the host in terms of the ground-state electron density in the unperturbed host and some coupling properties of the inserted impurity atom.
The latter can be calculated once and for all for any given atom, whereafter the electronic interaction terms are numerically easy to evaluate for any system where a reasonable description of the host electron density can be found. As will be discussed below, the perturbative interaction terms can be calculated relatively accurately for inert atoms, but the situation is less satisfactory for hydrogen. For the latter no systematic expansion of the interaction exists and the approach is partly guided by physical intuition. It must thus be remembered that while the potential-energy surfaces thus obtained are qualitatively correct, quantitative accuracy in the interaction energies is significant only at the level of about $0.2-0.5 \mathrm{eV}$. Nevertheless, the simplicity of the scheme makes further work in improving the description very much worthwhile: It makes it possible to investigate the trends in large classes of complicated systems where more rigorous approaches are at present all but impossible. The applications discussed so far include hydrogen heats of solution in transition metals, ${ }^{5}$ hydrogen at nickel $^{6}$ and other transition-metal ${ }^{7}$ surfaces, as well as hydrogen at defects in nickel, ${ }^{8}$ trapping of helium in metal vacancies, ${ }^{9}$ and helium surface scattering. ${ }^{10}$ Very recently, Daw and coworkers $^{11,12}$ have applied a related semiempirical scheme in their treatment of hydrogen embrittlement and hydrogen trapping to interstitial impurities.

The other aspect of light impurities interacting with solids has received much less attention in the form of quantitative calculations. A straightforward route to follow would be to describe the impurity-host and host-host interactions by (semi-) empirical pair potentials, and search for equilibrium relaxations using either computersimulation or lattice-response-function techniques. ${ }^{13}$ However, a pair-potential description is of limited use for metallic systems, where volume- or density-dependent terms in the total energy are important. Moreover, even if satisfactory pair potentials could be defined, these 
methods completely ignore the zero-point motion of the impurity, which can be important. An extreme example is the case of a positron, where the quantum kinetic energy dominates and dictates the basic features of diffusion and trapping at defects. In important recent publications, ${ }^{14,15}$ Sugimoto and Fukai have considered the quantum-mechanical nature of hydrogenic impurities in some bcc transition metals. Their approach is based on empirical metal-hydrogen pair potentials, but includes a proper account of the zero-point effects, which can be substantial. In another recent publication, Casella ${ }^{16}$ has suggested that the hydrogen states excited in neutron scattering are wave-mechanical band states, and shows this to be in accord with experimental findings. Further evidence for the quantum nature is provided by, e.g., the observation $^{17}$ of a tunnel-split oscillator ground state for hydrogen in oxygen-doped $\mathrm{Nb}$.

In this paper we seek to combine the electronic, latticerelaxation, and zero-point effects into a unified theory of light impurities in metals. We describe the electronic interaction in terms of the effective-medium theory, and calculate a potential-energy field of an impurity in a given host-ion configuration. In practice, this is facilitated by constructing the host electron density as a superposition of atomic densities. The Schrödinger equation for the impurity-mass coordinate is then solved numerically. The forces exerted on the host atoms by the impurity are calculated, and the ensuing lattice relaxations are evaluated by Green-function techniques. A new electron-density map and potential-energy field are then constructed, and the process is iterated to self-consistency. The main difference with the Sugimoto-Fukai approach ${ }^{14}$ is the potential-energy construction, which we carry out without any adjustable parameters. We present a number of applications for both bcc and fcc metals concerning the selftrapping of helium, hydrogen isotopes, and muons in the interstitial regions. We also discuss the excited-state characteristics and the diffusion mechanisms. While the main emphasis in this paper is on the laying out of the theoretical principles and assessing the potential and the deficiencies of the method, we also compare our results with existing experimental information. The overall performance and predictive power of the method are deemed good, but directions for further improvements are pointed out.

\section{FORMULATION}

We first briefly summarize the main formulas given by Sugimoto and Fukai ${ }^{14}$ since they also form our starting point. In the adiabatic approximation, the total energy of the combined light-impurity-metal system is

$$
E(\{\overrightarrow{\mathbf{u}}(\overrightarrow{\mathbf{R}})\}, \alpha)=E_{L}(\{\overrightarrow{\mathbf{u}}(\overrightarrow{\mathbf{R}})\})+E_{\alpha}(\{\overrightarrow{\mathbf{u}}(\overrightarrow{\mathbf{R}})\}),
$$

where $\{\overrightarrow{\mathbf{u}}(\overrightarrow{\mathbf{R}})\}$ is the set of the host-atom displacements at lattice positions $\overrightarrow{\mathrm{R}}, E_{L}$ is the (lattice) energy of the host atoms in the presence of the impurity, and $E_{\alpha}$ is the impurity energy eigenvalue. Fundamentally, all the energies in Eq. (1) are of electronic origin, but we assume in the spirit of the Born-Oppenheimer approximation that they can be reduced to a simpler representation in terms of well-defined coupling constants. The key equation is then the Schrödinger equation for the impurity coordinate,

$$
\left[-\frac{\hbar^{2}}{2 M_{\alpha}} \nabla^{2}+V(\overrightarrow{\mathrm{r}})\right] \psi_{\alpha}(\overrightarrow{\mathrm{r}})=E_{\alpha} \psi_{\alpha}(\overrightarrow{\mathrm{r}}),
$$

where $M_{\alpha}$ is the impurity mass and $V(\vec{r})$ is the potential-energy field "experienced" by the impurity atom. $V(\vec{r})$ arises from the "embedding" interaction between the impurity and host, and will be developed below in terms of the effective-medium theory. The practical details of the numerical solution of Eq. (2) are discussed in Appendix A.

In the harmonic solid approximation, the lattice energy $E_{L}$ is

$E_{L}=E_{0}-\sum_{\overrightarrow{\mathbf{R}}} \overrightarrow{\mathbf{F}}(\overrightarrow{\mathbf{R}}) \cdot \overrightarrow{\mathbf{u}}(\overrightarrow{\mathbf{R}})+\frac{1}{2} \sum_{\overrightarrow{\mathbf{R}}, \overrightarrow{\mathbf{R}}} \overrightarrow{\mathbf{u}}\left(\overrightarrow{\mathbf{R}}^{\prime}\right) \phi(\overrightarrow{\mathbf{R}}, \overrightarrow{\mathbf{R}}) \overrightarrow{\mathbf{u}}^{T}(\overrightarrow{\mathbf{R}})$,

where $E_{0}$ is the energy of the undeformed lattice, and $\vec{F}(\vec{R})$ is the force acting on the host atom at $\vec{R}$,

$$
F_{i}(\overrightarrow{\mathrm{R}})=-\left(\frac{\partial E_{L}}{\partial u_{i}(\overrightarrow{\mathrm{R}})}\right)_{0}, i=x, y, z
$$

The subscript 0 denotes that the derivative is evaluated at the equilibrium position in the undeformed crystal. Consequently, the forces vanish in the perfect lattice. $\underline{\phi}(\overrightarrow{\mathbf{R}}, \overrightarrow{\mathbf{R}})$ is the dynamic matrix,

$$
\phi_{i j}\left(\overrightarrow{\mathbf{R}}^{\prime}, \overrightarrow{\mathbf{R}}\right)=\left(\frac{\partial^{2} E_{L}}{\partial u_{i}\left(\overrightarrow{\mathbf{R}}^{\prime}\right) \partial u_{j}(\overrightarrow{\mathbf{R}})}\right]_{0} .
$$

The impurity (defect) brings in two features. The dynamic matrix changes to

$$
\underline{\phi}^{*}=\underline{\phi}-\delta \underline{\phi},
$$

and the forces cease to vanish. The equilibrium configuration corresponds to the variational minimum of Eq. (3) with respect to the displacements $\overrightarrow{\mathbf{u}}(\overrightarrow{\mathbf{R}})$, whereby ${ }^{18,19}$

$$
\overrightarrow{\mathbf{F}}(\overrightarrow{\mathbf{R}})=\sum_{\overrightarrow{\mathbf{R}}} \underline{\phi}^{*}\left(\overrightarrow{\mathbf{R}}, \overrightarrow{\mathbf{R}}^{\prime}\right) \overrightarrow{\mathbf{u}}^{T}\left(\overrightarrow{\mathbf{R}}^{\prime}\right) .
$$

The inverse of Eq. (7) reads

$$
\overrightarrow{\mathbf{u}}(R)=\sum_{\overrightarrow{\mathbf{R}}} \underline{G}^{*}\left(\overrightarrow{\mathbf{R}}, \overrightarrow{\mathbf{R}}^{\prime}\right) \overrightarrow{\mathrm{F}}^{T}(\overrightarrow{\mathbf{R}}),
$$

where $G^{*}$ is the static Green function for the deformed lattice. ${ }^{19} G_{i j}^{*}\left(\vec{R}, \vec{R}^{\prime}\right)$ gives the displacement in the direction $i$ for the atom at $\overrightarrow{\mathbf{R}}$ when a unit force in the direction $j$ is exerted on the atom at $\vec{R}^{\prime}$. Combining (6) and (8), one has (in matrix notation)

$$
\overrightarrow{\mathrm{u}}=\underline{G}(\overrightarrow{\mathrm{F}}+\delta \underline{\overrightarrow{\mathrm{u}}})=\underline{G} \overrightarrow{\mathrm{F}}^{*},
$$

where $\underline{G}$ is the perfect-lattice Green function $\left(\underline{G}=\phi^{-1}\right)$, and $\overrightarrow{\mathrm{F}}^{*}$ is the Kanzaki force ${ }^{20}$ exerted by the defect on a lattice atom in the relaxed position. The latticedeformation energy is now expressed simply as 


$$
\Delta E_{L}=E_{L}-E_{0}=\frac{1}{2} \sum_{\overrightarrow{\mathrm{R}}} \overrightarrow{\mathrm{F}}^{*}(\overrightarrow{\mathrm{R}}) \cdot \overrightarrow{\mathrm{u}}(\overrightarrow{\mathrm{R}}) .
$$

Finally, the Kanzaki forces can be obtained from the impurity distribution $\left|\psi_{\alpha}\right|^{2}$ and the potential-energy field $V$. The latter is a function of the atomic positions. For an atom at the relaxed position $\vec{R}+\overrightarrow{\mathbf{u}}(\overrightarrow{\mathbf{R}})$,

$\overrightarrow{\mathbf{F}}^{*}(\overrightarrow{\mathbf{R}})=-\int d \overrightarrow{\mathbf{r}}\left|\psi_{\alpha}(\overrightarrow{\mathbf{r}})\right|^{2} \frac{\partial V(\ldots,|\overrightarrow{\mathbf{R}}+\overrightarrow{\mathbf{u}}(\overrightarrow{\mathbf{R}})-\overrightarrow{\mathbf{r}}|, \ldots)}{\partial|\overrightarrow{\mathbf{R}}+\overrightarrow{\mathbf{u}}(\overrightarrow{\mathbf{R}})-\overrightarrow{\mathbf{r}}|}$.

The Green function for the ideal discrete lattice can be written as ${ }^{18}$

$G_{i j}(\overrightarrow{\mathrm{R}})=\frac{\Omega}{(2 \pi)^{3}} \sum_{\sigma} \int_{\mathrm{BZ}} d \overrightarrow{\mathrm{q}} e_{i}^{\sigma}(\overrightarrow{\mathrm{q}}) e_{j}^{\sigma}(\overrightarrow{\mathrm{q}}) \frac{e^{-i \overrightarrow{\mathrm{q}} \cdot \overrightarrow{\mathrm{R}}}}{\omega_{\sigma}^{2}(\overrightarrow{\mathrm{q}})}$,

where $\Omega$ is the unit-cell volume, $e_{i}^{\sigma}(\overrightarrow{\mathrm{q}})(\sigma=1,2,3)$ are the eigenvectors, and $\omega_{\sigma}^{2}$ are the corresponding eigenvalues of the matrix $\underline{\phi}^{-1}(\overrightarrow{\mathrm{q}})$ when

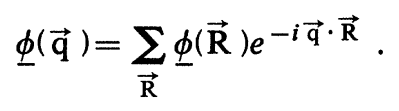

In other words, Eq. (12) contains the Brillouin-zone (BZ) integration over the inverse squares of the phonon modes $\omega_{\sigma}(\vec{q})$ multiplied by the corresponding polarization vectors $e_{i}^{\sigma}(\overrightarrow{\mathrm{q}})$.

The discrete-lattice Green functions are numerically available for most metals of interest. ${ }^{19,21}$ In cubic crystals it is straightforward to also derive the following elastic continuum approximations: ${ }^{22}$

$$
\phi_{i j}(\overrightarrow{\mathrm{q}})=\Omega \sum_{k, l} c_{i k j l} q_{k} q_{l},
$$

with

$$
\begin{aligned}
c_{i k j l}= & c_{12} \delta_{i k} \delta_{j l}+c_{44}\left(\delta_{i j} \delta_{k l}+\delta_{i l} \delta_{j k}\right) \\
& +\left(c_{11}-c_{12}-2 c_{44}\right) \delta_{i j k l} .
\end{aligned}
$$

Above $c_{11}, c_{12}$, and $c_{44}$ are the (Voigt) elastic constants, and $\delta_{i j k l}=1$ if $i=j=k=l$, or $\delta_{i j k l}=0$ otherwise. It follows for the elastic Green function that

$$
G_{i j}(\overrightarrow{\mathbf{R}})=\frac{1}{8 \pi^{2} R} \int_{0}^{2 \pi} \gamma_{i j}(\varphi) d \varphi,
$$

where

$$
\begin{aligned}
\gamma_{i j}(\varphi)=\frac{\delta_{i j}}{c_{44}+d \kappa_{j}}-\frac{\kappa_{i} \kappa_{j}\left(c_{44}+c_{12}\right)}{\left(c_{44}+d \kappa_{i}\right)\left(c_{44}+d \kappa_{j}^{2}\right)\left(1+\sum_{k=1}^{3} \frac{c_{44}+c_{12}}{c_{44}+d \kappa_{k}^{2}} \kappa_{k}^{2}\right)}, \\
\quad d=c_{11}-c_{12}-2 c_{44}, \quad \kappa_{1}=\cos \varphi \sin \phi+\sin \varphi \cos \theta \cos \phi, \quad \kappa_{2}=-\cos \varphi \cos \phi+\sin \varphi \cos \theta \sin \phi, \kappa_{3}=\sin \varphi \sin \theta,
\end{aligned}
$$

and $\theta$ and $\phi$ are the polar angles of $\vec{R}$. The integral in Eq. (16) can be carried out analytically only in a few main symmetry directions, but is easily obtained numerically in a general direction. The Green function (16) diverges at the origin, but the $G_{11}(0)$ component needed in the calculations can be evaluated in the Debye model, in which this divergence does not exist. ${ }^{19}$ The final form in the elastic continuum model reads as

$$
G_{11}(0)=\frac{\left(c_{11}+2 c_{44}\right)^{1 / 2} q_{D}}{2 \pi^{2} c_{44}\left(c_{11}\right)^{1 / 2}},
$$

where $q_{D}$ is the Debye wave vector. The agreement between the elastic and discrete-lattice Green functions is normally better than $\sim 10 \%$. Larger deviations occur for metals with long-range elastic coupling, of which $\mathrm{Nb}$ is a typical example. ${ }^{23}$

In practice, only few (in our calculations, two) nearestneighbor atom shells around the impurity are allowed to relax. This limitation and the use of symmetry reduces the dimension of the Green-function matrix considerably. ${ }^{19}$ The expressions for the resulting reduced Green functions for the tetrahedral and octahedral interstices in both bcc and fcc lattices are given in Appendix B.

Equations (2), (9), and (11) constitute a set of equations which has to be solved self-consistently. What remains is a description of the interaction energy $V$. We utilize the quasiatom concept. ${ }^{3-5}$ First, we assume that a satisfactory approximation for the electron density $n_{0}(\vec{r})$ in the host (without the impurity, but with atomic relaxations) is available. We further suppose that the impurity nucleus is placed at the position $\vec{r}=\overrightarrow{0}$. The electronic perturbation due to the impurity atom is, owing to metallic screening, localized in space. We assume that the impurityinduced effects are confined within a sphere of cutoff radius $R_{a}$. Inside this sphere the charge density $\rho_{a}(\overrightarrow{\mathrm{r}})$ and Coulomb potential $\phi_{a}(\vec{r})$ are taken equal to those for an atom embedded in a homogeneous electron gas. ${ }^{24}$ The density $\bar{n}_{0}$ where the embedding is imagined to take place is chosen by using $\phi_{a}(\vec{r})$ as the sampling function for the unperturbed host,

$$
\bar{n}_{0}=\int_{R_{a}} d \overrightarrow{\mathrm{r}} \phi_{a}(\overrightarrow{\mathrm{r}}) n_{0}(\overrightarrow{\mathrm{r}}) / \int_{R_{a}} d \overrightarrow{\mathrm{r}} \phi_{a}(\overrightarrow{\mathrm{r}}) .
$$

This implies a self-consistency requirement for $\bar{n}_{0}$ and $\phi_{a}(\overrightarrow{\mathrm{r}})$. In practice, this is not very severe since the atominduced Coulomb potential $\phi_{a}$ is a weak function of the (jellium) density where it is calculated. For further development, it is important that the quasiatom is neutral inside $R_{a}$, and also that $R_{a}$ is smaller than any impurityhost nuclear separation. Because of long-range Friedel oscillations, this, in practice, necessitates a renormalization of the positive charge (see Sec. III A).

With these assumptions, the interaction energy $V$ between the impurity at $\vec{r}=\overrightarrow{0}$ and the host [electron density $\left.n_{0}(\overrightarrow{\mathbf{r}})\right]$ can be written as a perturbation series,

$$
\Delta E_{\mathrm{hom}}\left(\bar{n}_{0}\right)-\alpha_{a} \bar{n}_{0}+\delta \sum_{i} \Delta \epsilon_{i}
$$

where $\Delta E_{\text {hom }}\left(n_{0}\right)$ is the immersion energy ${ }^{24}$ of an atom 
into a homogeneous electron gas of density $n_{0}$, and

$\alpha_{a}=-\int_{R_{a}} d \overrightarrow{\mathrm{r}} \phi_{a}(\overrightarrow{\mathrm{r}})=-\int_{R_{a}} \int_{R_{a}} d \overrightarrow{\mathrm{r}} d \overrightarrow{\mathrm{r}}^{\prime} \frac{\rho_{a}(\overrightarrow{\mathrm{r}})}{\left|\overrightarrow{\mathrm{r}}-\overrightarrow{\mathrm{r}}^{\prime}\right|}$

is proportional to the mean quasiatomic Coulomb potential. The last term in Eq. (20) denotes the change in the one-electron eigenvalue differences when the impurityjellium system is replaced by the impurity-true-host system,

$$
\begin{aligned}
\delta \sum_{i} \Delta \epsilon_{i}= & {\left[\sum_{i} \epsilon_{i}\right]_{\text {host-imp }}-\left[\sum_{i} \epsilon_{i}\right]_{\text {host }} } \\
& -\left[\sum_{i} \epsilon_{i}\right]_{\text {jell-imp }}+\left[\sum_{i} \epsilon_{i}\right]_{\text {jell }} .
\end{aligned}
$$

The feasibility of the effective-medium theory as outlined above demands that (i) the intrinsic dependence on the cutoff radius $R_{a}$ in Eq. (20) should be small for reasonable values of $R_{a}$, and (ii) one should be able to estimate the one-electron term (22) without excessive effort (that is, certainly short of performing the full calculation of the energy spectrum in the combined host-impurity system). We shall examine these questions for hydrogen and helium in detail below.

In all the applications described below we have simply constructed the host electron density by superimposing free-atom (at) densities, i.e.,

$$
n_{0}(\overrightarrow{\mathrm{r}})=\sum_{\overrightarrow{\mathbf{R}}_{i}} n_{\mathrm{at}}\left(\overrightarrow{\mathrm{r}}-\overrightarrow{\mathbf{R}}_{i}\right),
$$

where the sum goes over the atomic positions. For the present purposes, this approximation is accurate enough: Comparison with available self-consistent calculations ${ }^{25}$ shows that the differences in the interstitial regions are less than about $10 \%$ for close-packed metallic elements. For vacancies and other lattice defects, differences may become larger but the qualitative features are not changed.

\section{HYDROGEN IN METALS}

\section{A. Hydrogen effective-medium potential}

In the case of hydrogen the covalent term $\delta \sum_{i} \Delta \epsilon_{i}$ of the effective-medium potential ${ }^{20}$ is important because the hydrogen $1 s$ level interacts strongly with the host valence bands. Therefore the situation is completely different from that for helium, where the tightly bound $1 s$ level is nearly inert, also in a metallic environment (see Sec. IV). In transition metals the hybridization of the hydrogen $1 \mathrm{~s}$ and host $d$ levels occurs, and the covalent contribution can approximately be expressed as a sum of two terms, ${ }^{5}$

$$
\begin{aligned}
\delta \sum_{i} \Delta \epsilon_{i}= & \int_{R_{a}}\left[n_{0}(\overrightarrow{\mathrm{r}})-\bar{n}_{0}\right] \Delta V_{a}(\overrightarrow{\mathrm{r}}) d \overrightarrow{\mathrm{r}} \\
& -2(1-f) 20 \frac{\Delta_{d} \Delta_{a}}{C_{d}-V^{0}} \sum_{\overrightarrow{\mathrm{R}}_{i}} \frac{1}{\left|\overrightarrow{\mathrm{r}}-\overrightarrow{\mathrm{R}}_{i}\right|^{6}},
\end{aligned}
$$

where $\Delta V_{a}(\overrightarrow{\mathrm{r}})$ is the total effective potential change due to the hydrogen. The second term is derived in the atomicsphere approximation (ASA). ${ }^{26}$ In Eq. (24), $f$ is the relative filling of host $d$ level, $C_{d}-V^{0}$ is the separation between the $d$-band center of gravity and the metal effective potential at the hydrogen site, $\Delta_{a}$ and $\Delta_{d}$ are functions of the potential and atomic radii in ASA, and finally the sum in the equation goes over all host lattice sites.

As suggested by Nørskov, ${ }^{5}$ we have chosen the cutoff radius $R_{a}=2.5 a_{0}$ for hydrogen-induced density and potential. The induced density is calculated by embedding hydrogen in a uniform electron gas with a density parameter $r_{s}=3 a_{0}$. We also follow the recommendation of Ref. 7 and renormalize the charge inside the sphere determined by this radius by adding a uniform neutralizing background charge distribution. Also, as recommended, ${ }^{7} \Delta V_{a}$ is rigidly shifted so that it vanishes at the surface of the renormalization sphere. When these instructions are followed, the effective-medium potential is relatively insensitive to reasonable variations in the cutoff radius $R_{a}$. On the other hand, a different procedure, for example, the renormalization of the hydrogen nuclear charge, leads to a severe dependence on the cutoff radius.

In the actual calculations we have omitted the small second (hybridization) term in the covalent contribution [Eq. (24)] and used Nørskov's ${ }^{5}$ rough estimate for the first term

$$
\int_{R_{a}}\left[n_{0}(\overrightarrow{\mathrm{r}})-\bar{n}_{0}\right] \Delta V_{a}(\overrightarrow{\mathrm{r}}) d \overrightarrow{\mathrm{r}}=-\alpha_{v} \bar{n}_{0},
$$

where $\alpha_{v}=31 \mathrm{eV} a_{0}^{3}$. These approximations are made because we want to keep the construction of the potential as simple as possible, and the determination of the hybridization term appears somewhat arbitrary against the background of approximations made in calculating the leading contributions to the potential. For the same reason, we have not included the recently suggested correction ${ }^{7}$ which takes into account the orthogonality repulsion between hydrogen and metal-ion cores. However, these omissions are not severe, because, as emphasized by Nørskov, ${ }^{5}$ the hybridization and repulsion terms are only small corrections to the potential energy, introduced in order to get the energy minima to agree with such experimental results as the heat of solution or the binding energy of hydrogen chemisorbed on metal surfaces. The leading contributions, which we keep, determine, in turn, the trends and the spatial variation of the potential, which are essential in our application. Thus, we calculate the potential for hydrogen in the host from the sampled density [Eq. (19)] corresponding to a given point using the following interpolation formulas ( $n_{0}$ in $a_{0}^{-3}$ and $\Delta E_{\text {hom }}$ in $\mathrm{eV}$ ):

$$
\begin{aligned}
& V(\overrightarrow{\mathrm{r}})=\Delta E_{\mathrm{hom}}\left[\bar{n}_{0}(\overrightarrow{\mathrm{r}})\right]-\alpha_{\mathrm{at}} \bar{n}_{0}(\overrightarrow{\mathrm{r}})-\alpha_{v} \bar{n}_{0}(\overrightarrow{\mathrm{r}}) \\
& \Delta E_{\mathrm{hom}}\left(n_{0}\right)= \begin{cases}130 n_{0} \ln \left(\frac{n_{0}}{0.004}\right)-133 n_{0}-1.12, & 0.002 \leq n_{0}<0.0127 \\
398\left(n_{0}-0.0 .127\right)^{2}+150 n_{0}-2.81, & 0.0127 \leq n_{0} .\end{cases}
\end{aligned}
$$


TABLE I. Dependence of the hydrogen effective-medium potential on the cutoff radius $R_{a}$. Hydrogen is embedded in the unrelaxed octahedral site in $\mathrm{Cu} . \bar{n}_{0}$ and $\alpha_{a}$ are defined in Eqs. (19) and (21), respectively. $\quad V$ is calculated via Eq. (26).

\begin{tabular}{lccc}
\hline \hline $\begin{array}{c}R_{a} \\
\left(a_{0}\right)\end{array}$ & $\begin{array}{c}\bar{n}_{0} \\
\left(a_{0}^{-3}\right)\end{array}$ & $\begin{array}{c}\alpha_{a} \\
\left(\mathrm{eV} a_{0}^{3}\right)\end{array}$ & $\begin{array}{c}V \\
(\mathrm{eV})\end{array}$ \\
\hline 2 & 0.0212 & 85.3 & -2.07 \\
2.3 & 0.0221 & 93.3 & -2.21 \\
2.5 & 0.0224 & 95.1 & -2.24 \\
2.7 & 0.0221 & 94.1 & -2.18 \\
\hline \hline
\end{tabular}

Nørskov's values, ${ }^{5} \alpha_{a}=88 \mathrm{eV} a_{0}^{3}$ and $\alpha_{v}=31 \mathrm{eV} a_{0}^{3}$, are used.

We have tested the stability of the effective-medium potential against the variations of the cutoff radius $R_{a}$ by embedding the hydrogen atom in the octahedral site in unrelaxed $\mathrm{Cu}$ lattice. The results are shown in Table I. The values for $\alpha_{a}$ and $\bar{n}_{0}$ are slightly different from those represented by Nørskov ${ }^{5}$ and have maxima at $R_{a} \approx 2.5 a_{0}$. Consequently, the effective-medium potential is also fairly insensitive to the cutoff radius in this region. The increase of $R_{a}$ above $\approx 2.7 a_{0}$ gives rise to difficulties because of the Friedel oscillations overlapping with the $\mathrm{Cu}$ core region. In these calculations $\alpha_{v}$ is kept constant because the changes in the potential due to the variation in $\alpha_{v}$ are canceled by the changes in the repulsive orthogonality contribution, ${ }^{27}$ which we have omitted in our approach. As will be discussed below, the effective-medium potential of Eq. (26) gives a good description near the minima, but is probably too soft near the host-ion cores.

\section{B. bcc metals}

Figure 1 demonstrates the self-trapping phenomena for hydrogen in the octahedral site of bcc $\mathrm{Nb}$ according to our model. The figure shows the potential and wave function along the line joining the neighboring octahedral and tetrahedral sites. The upper solid and dashed curves give the wave function and potential, respectively, for the delocalized band state. The wave function has maxima at the equivalent tetrahedral sites and it vanishes at the octahedral sites. The two lower curves refer to the self-trapped state. In the self-trapped state, hydrogen has deformed the host lattice symmetrically around the octahedral site and caused the lowering of the potential into a local potential well. The hydrogen wave function is relatively flat in the self-trapped state and has its maximum at the octahedral point. Therefore in this model we shall consider this state as being self-trapped into the octahedral interstice rather than as being a tunneling state between the four equivalent tetrahedral interstices around the octahedral site. The energy levels of the delocalized and trapped states are also indicated in Fig. 1. The potential deformation associated with the self-trapping lowers the energy level remarkably. The self-trapping process becomes favorable if this lowering is greater in magnitude than the energy paid for the lattice deformation.

Figure 2 shows the hydrogen wave function in two di-

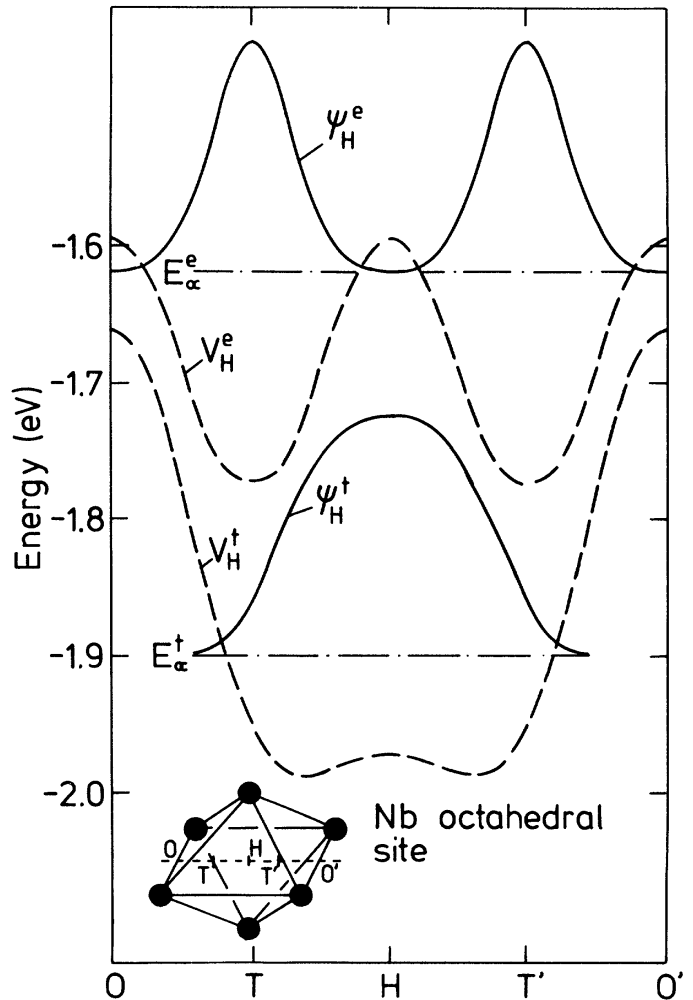

FIG. 1. Self-trapping of hydrogen into the octahedral site in $\mathrm{Nb}$. The upper solid and dashed curves give, respectively, the wave function $\psi_{\mathrm{H}}^{e}$ and the potential $V_{\mathrm{H}}^{e}$ for the delocalized band state along the line $O O^{\prime}$ given in the inset. The lower curves $\psi_{\mathrm{H}}^{t}$ and $V_{\mathrm{H}}^{t}$ give the corresponding functions for the self-trapped state. The dashed-dotted lines $E_{\alpha}^{e}$ and $E_{\alpha}^{t}$ indicate the absolute energy levels for hydrogen in the band state and the self-trapped state, respectively.

mensions on the plane of the four second-nearest neighbors of the octahedral site. The contour plot clearly expresses the importance of the zero-point motion for the light hydrogen. The self-trapping process into the tetrahedral site of $\mathrm{Nb}$ is shown in Fig. 3. The extent of the wave function is of the same order as in the octahedral

\section{$\mathrm{Nb}$ octahedral site}
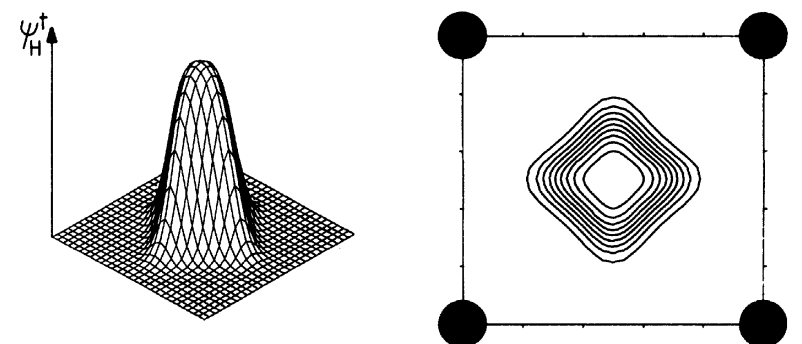

FIG. 2. Hydrogen wave function self-trapped in the octahedral site in $\mathrm{Nb}$. The second-nearest neighbors are denoted by solid circles. The contour spacing in the contour plot is onetenth of the maximum value. 


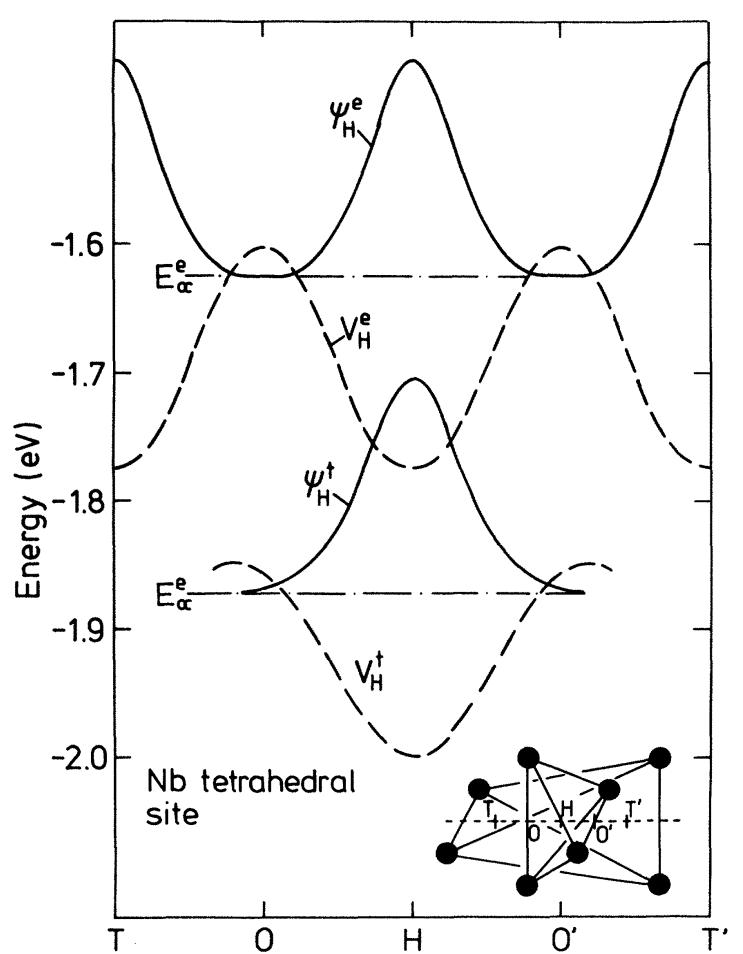

FIG. 3. Self-trapping of hydrogen into the tetrahedral site in $\mathrm{Nb}$. For details, see the caption of Fig. 1.

site, but now the wave function is more peaked due to the steeper potential well.

The formation of the self-trapping well in the case of the octahedral site in bcc metals is mainly due to the relaxation of the two first-nearest-neighbor host ions. Ac- cording to Table II, which lists our results for the relaxed ion positions for the first-nearest and second-nearest neighbors of hydrogen in the octahedral and tetrahedral sites of various bcc metals, the first-nearest-neighbor distance increases about $7-11 \%$ in the presence of hydrogen. Around the tetrahedral site the relaxation of the four first-nearest neighbors is less, about $4-7 \%$. In both cases the relaxation of the second-nearest neighbors is minor compared with the first-nearest neighbors. The secondnearest neighbors can even move inwards. For $\mathrm{V}$ and $\mathrm{Ta}$ the calculations are performed by using both the elastic and the discrete-lattice Green functions. The relaxations depend slightly on the choice of the response function, but the characteristic differences between octahedral and tetrahedral sites are similar at least for the first-nearest neighbor; for the energetics, the relaxation of the secondnearest neighbor is unimportant due to its small magnitude. Our results for the relaxation are somewhat smaller than the values obtained by Sugimoto and Fukai, ${ }^{14}$ and the experimental results of Behr et al. ${ }^{28}$ Sugimoto and Fukai obtain, with the use of a Born-Mayer-type potential, relaxations of $6.1 \%$ and $8.2 \%$ for the first-nearest neighbors of tetrahedral and octahedral hydrogen in $\mathrm{Nb}$, respectively. Behr et al. ${ }^{28}$ conclude from the static Debye-Waller factors of x-ray measurements that hydrogen induces the first-nearest neighbor relaxations of $8.9 \%$, $6.1 \%$, and $4.6 \%$ around the tetrahedral site in $\mathrm{V}, \mathrm{Nb}$, and $\mathrm{Ta}$, respectively. This comparison seems to reflect the softness of the effective-medium potential.

The results concerning the energetics of hydrogen in the vanadium-group metals, and in $\mathrm{Cr}$ and $\mathrm{Fe}$, are collected in Table II. The prominent feature is that the self-trapping energies are nearly equal for the tetrahedral and octahe-

TABLE II. Calculated properties of hydrogen self-trapped in octahedral $(O)$ or tetrahedral $(T)$ sites in bcc metals. $L$ refers to the Green functions of discrete-lattice theory and $E$ refers to the elastic Green functions. The former are taken from Ref. 19 and the latter are calculated using the elastic data collected in Ref. 18. The relaxation is defined as the percentage change in the interstitialsite-atom distance. $\Delta E_{\alpha}$ is the change in the absolute energy level between the band state and the self-trapped state. $E_{L}$ is the energy stored in the lattice relaxation. $E_{0}$ is the zero-point energy defined as the distance between the energy level and the minimum of the potential. $E_{\mathrm{ST}}=\Delta E_{\alpha}+E_{L}$ is the energy gain in self-trapping.

\begin{tabular}{|c|c|c|c|c|c|c|c|c|}
\hline \multirow[b]{2}{*}{ Metal } & \multirow[b]{2}{*}{ Site } & \multirow{2}{*}{$\begin{array}{l}\text { Green } \\
\text { function }\end{array}$} & \multirow{2}{*}{$\begin{array}{l}\Delta E_{\alpha} \\
(\mathrm{eV})\end{array}$} & \multirow{2}{*}{$\begin{array}{c}E_{L} \\
(\mathrm{eV})\end{array}$} & \multirow{2}{*}{$\begin{array}{c}E_{0} \\
(\mathrm{eV})\end{array}$} & \multirow{2}{*}{$\begin{array}{l}E_{\mathrm{ST}} \\
(\mathrm{eV})\end{array}$} & \multicolumn{2}{|c|}{ Displacements $(\%)$} \\
\hline & & & & & & & First neighbors & Second neighbors \\
\hline \multirow[t]{4}{*}{$\mathbf{V}$} & 0 & $L$ & -0.322 & 0.145 & 0.118 & -0.177 & 9.3 & -0.2 \\
\hline & $T$ & $L$ & -0.303 & 0.123 & 0.150 & -0.180 & 4.6 & -0.5 \\
\hline & 0 & $\boldsymbol{E}$ & -0.428 & 0.169 & 0.109 & -0.259 & 11.7 & 1.4 \\
\hline & $T$ & $E$ & -0.420 & 0.152 & 0.129 & -0.268 & 7.1 & -0.1 \\
\hline \multirow[t]{2}{*}{$\mathrm{Nb}$} & $O$ & $L$ & -0.264 & 0.125 & 0.107 & -0.139 & 6.9 & 0.2 \\
\hline & $T$ & $L$ & -0.256 & 0.105 & 0.129 & -0.151 & 4.1 & -0.4 \\
\hline \multirow[t]{4}{*}{$\mathrm{Ta}$} & $O$ & $L$ & -0.305 & 0.147 & 0.113 & -0.158 & 7.6 & 0.2 \\
\hline & $T$ & $L$ & -0.295 & 0.123 & 0.137 & -0.172 & 3.9 & -0.2 \\
\hline & $O$ & $E$ & -0.343 & 0.159 & 0.111 & -0.184 & 8.0 & 0.2 \\
\hline & $T$ & $E$ & -0.349 & 0.141 & 0.134 & -0.208 & 4.8 & -0.1 \\
\hline \multirow[t]{2}{*}{$\mathrm{Cr}$} & $O$ & $E$ & -0.632 & 0.249 & 0.139 & -0.383 & 11.5 & 1.6 \\
\hline & $T$ & $E$ & -0.630 & 0.222 & 0.173 & -0.408 & 7.1 & 0.1 \\
\hline \multirow[t]{2}{*}{$\mathrm{Fe}$} & $O$ & $E$ & -0.622 & 0.252 & 0.156 & -0.370 & 11.5 & -0.2 \\
\hline & $T$ & $E$ & -0.589 & 0.223 & 0.185 & -0.365 & 5.8 & -0.5 \\
\hline
\end{tabular}




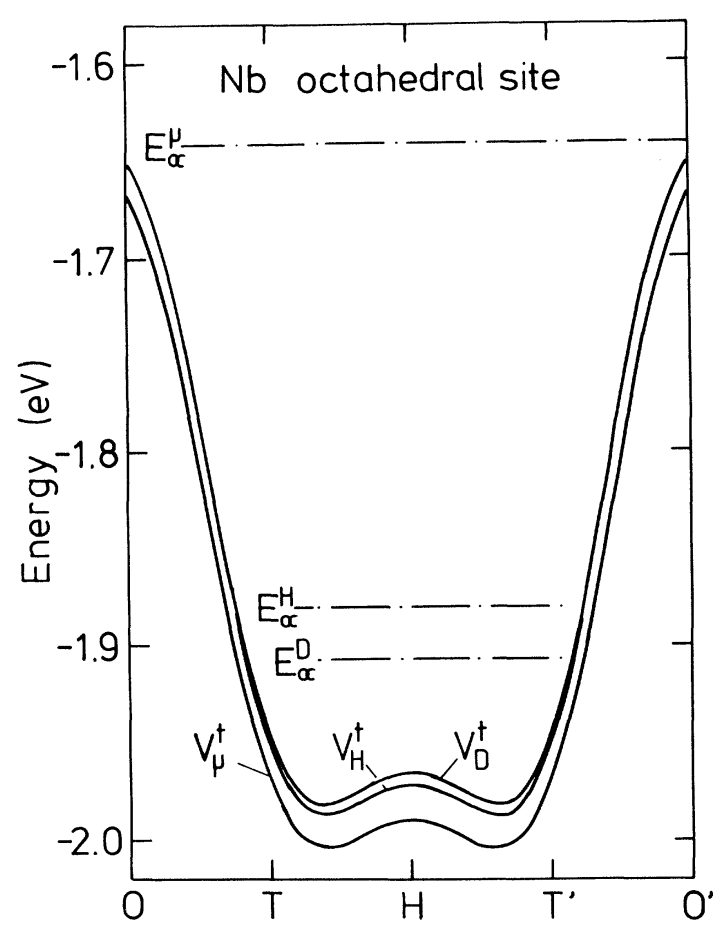

FIG. 4. Self-trapping potentials for hydrogenic impurities in the octahedral site in $\mathrm{Nb}$. The line $O O^{\prime}$ joins the octahedral sites in the $\langle 100\rangle$ direction (see the inset of Fig. 1). The energy eigenvalues for $\mu^{+}, H$, and $D$ are indicated by dashed-dotted lines.

dral locations, the tetrahedral site being slightly more favorable (except in $\mathrm{Fe}$ ). This degeneracy within $\sim 0.02$ $\mathrm{eV}$ is significant because the potential difference between these sites in the unrelaxed lattice is much larger $(0.2-0.4$ $\mathrm{eV}$ ) in these metals according to the quasiatom model. The reason for the degeneracy is that the lowering of the energy eigenvalue $\Delta E_{\alpha}$ and the energy $E_{L}$ stored in the lattice deformation are nearly equal for both sites. The energy-level change $\Delta E_{\alpha}$ is larger in magnitude for the octahedral site, but the smaller relaxation energy $E_{L}$ for the tetrahedral site makes it the lowest-energy site for hydrogen. According to Table II the replacement of the Green functions of the discrete-lattice theory by the elastic approximations changes the energy terms, but again the near degeneracy and the important differences between the octahedral and tetrahedral sites remain the same. Nørskov ${ }^{5}$ has calculated the hydrogen heat of solution in the $3 d$ transition metals using the effectivemedium theory, but ignoring the lattice relaxation and the hydrogen zero-point energy. The lowering of the potential due to the relaxation is of the same order as the sum of lattice distortion and zero-point energies. Thus, as a result, the inclusion of these effects changes the heat of solution typically less than $0.1 \mathrm{eV}$.

Figures 4 and 5 show, in one special direction, the effects of the isotope mass on the potential and the wave function in the localized octahedral state in $\mathrm{Nb}$. A positive muon $\mu^{+}$(mass equal to one-ninth of the proton mass) produces a significantly stronger relaxation than the hydrogen isotopes $\mathbf{H}$ or $\mathbf{D}$ due to its larger zero-point amplitude. Figure 4 also shows that the zero-point energies

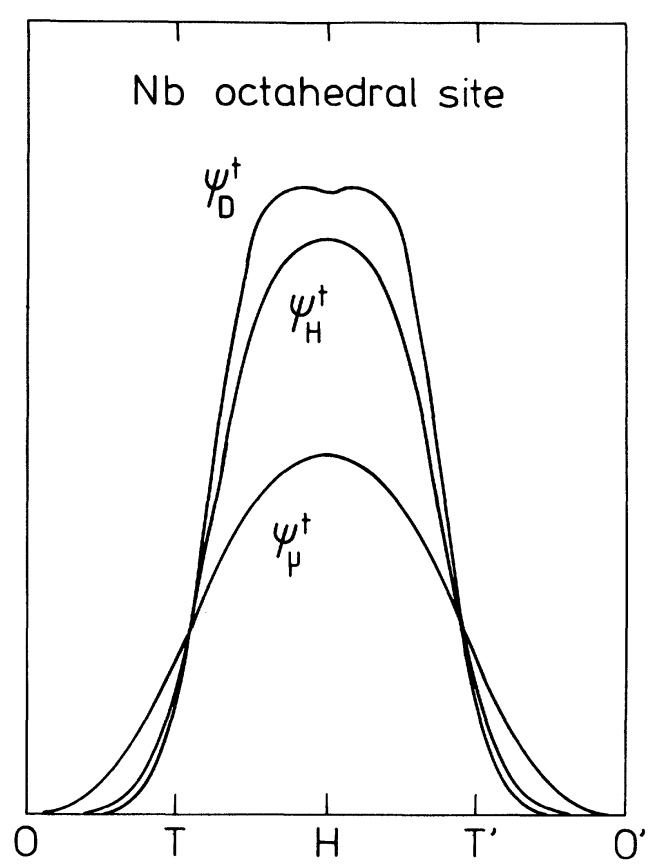

FIG. 5. Localized wave functions for hydrogenic impurities in the octahedral site in $\mathrm{Nb}$. The axis $O O^{\prime}$ is shown in the inset of Fig. 1.

of $\mathbf{H}$ and $\mathrm{D}$ are comparable, but the zero-point energy of $\mu^{+}$is much higher. The increase of the mass causes the wave functions in Fig. 5 to be more peaked. Owing to its lower zero-point energy, D "feels" the details of the potential better than $\mathbf{H}$, which is reflected in the wave function for $D$ as a weak local minimum at the octahedral point. However, the potential is not sufficiently strong to split the wave function into the tetrahedral regions.

Numerical results concerning the hydrogen-isotope effects in $\mathrm{Nb}$ are collected in Table III. The relaxation displacements of the first- and second-nearest neighbors saturate relative rapidly as the isotope mass increases. The influence of the mass in the energy terms is interesting. According to the self-trapping energies, the octahedral site turns out to be the stable one for $\mu^{+}$, contrary to the case in the hydrogen isotopes. The origin of this difference is that for $\mu^{+}$the deformation work in the tetrahedral site is nearly as large as in the octahedral site due to the large range of the wave function, but the deformation does not lower the potential and the energy level $E_{\alpha}$ to the same extent. The self-trapping energy gain decreases as the mass of the impurity increases, but at the same time the difference between the self-trapping energies corresponding to octahedral and tetrahedral sites increases. This point is important for the diffusion mechanism of hydrogen, as will be discussed below. It should be also noted that this energy difference saturates between $D$ and $T$ when the atom begins to resemble a "classical" point mass without zero-point motion.

Experimentally, the location of hydrogen in bcc metals has been investigated by ion-channeling techniques. ${ }^{29}$ The results indicate both tetrahedral and octahedral occupan- 
TABLE III. Isotope effects for hydrogenic impurities in $\mathrm{Nb} . E_{\alpha}$ is the energy eigenvalue with respect to vacuum, $E_{0}$ is the kinetic zero-point energy, $E_{L}$ is the lattice-deformation energy, and $E_{\mathrm{ST}}=\Delta E_{\alpha}+E_{L}$ is the self-trapping energy. $O$ refers to the octahedral site and $T$ refers to the tetrahedral site. The Green function of the discrete-lattice theory is used (Ref. 19). All energies are in eV.

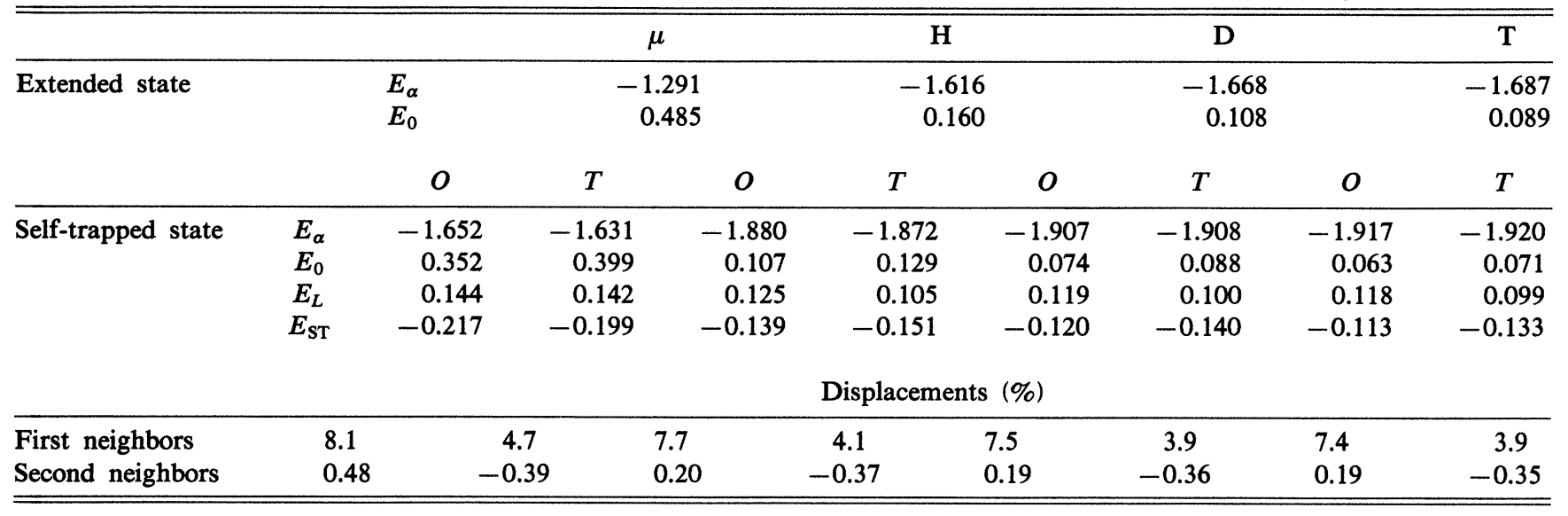

cy, depending on the host metal in question. In $\mathrm{Nb}, \mathrm{Ta}$, and $\mathrm{V}$ the assignment is the tetrahedral site, whereas in $\mathrm{Cr}$, which has a relatively small lattice constant, the octahedral location is found to be more probable. In V, both sites are possible according to the experiments. Our calculations are in agreement with these experiments, except for Cr. Table III shows that the tetrahedral site should be clearly more favorable than the octahedral one in $\mathrm{Cr}$. The interpretation of the ion-channeling experiments can also be influenced to some extent by the lattice defects generated during the implantation of the probe ions. The trapping of hydrogen by heavier impurities might also result in a location different from the one in the impurity-free lattice. $^{29}$ However, the possibility of both tetrahedral and octahedral locations of $\mathbf{H}$ indicates that these interstices are generally nearly degenerate in energy, also according to the experiments. From the muon experiments it is difficult to determine the stable location due to the strong muon-impurity interaction, and no reliable results have yet been found. ${ }^{1}$

\section{Diffusion model for hydrogen in bcc metals}

Because the self-trapping energies for hydrogen corresponding to tetrahedral and octahedral sites are nearly equal, it is tempting to assume (i) that the coherent tunneling region for the diffusion of hydrogen in bec metals occurs along the path connecting adjacent tetrahedral and octahedral sites, and (ii) hydrogen is capable of deforming the surrounding lattice continuously at every point on the diffusion path. This same conclusion has also recently been drawn by Emin, ${ }^{30}$ who based his investigation on the small-polaron concept. The activation energy for this kind of process should be of the same order of magnitude as the difference between the self-trapping energies for octahedral and tetrahedral sites, i.e., according to Table III, approximately $0.01-0.02 \mathrm{eV}$. These are less than the experimental values, $0.05-0.1 \mathrm{eV} .{ }^{31}$ The smalless of the calculated value is mainly due to the underestimation of the short-range orthogonality repulsion experienced by hydrogen near the host-metal nuclei. The short-range part of the metal-hydrogen interaction is important for the excitation energies. ${ }^{14}$ On the other hand, the effectivemedium potential predicts that the self-trapping energies are, in every case, much higher than the experimental activation energies. Thus during the activation process hydrogen cannot be in a state which extends over several lattice interstices, but the hydrogen wave function has to be relatively well localized.

Furthermore, the comparison of calculated and experimental results for the isotope effects confirms the "continuous-lattice-deformation" diffusion model. Namely, both the experimental ${ }^{31}$ high-temperature activation energies and the difference between the calculated octahedral- and tetrahedral-site self-trapping energies increase as the isotope mass increases. For example, the experimental $^{31}$ activation energies for $\mathrm{H}, \mathrm{D}$, and $\mathrm{T}$ in $\mathrm{Nb}$ are $0.106,0.127$, and $0.135 \mathrm{eV}$, respectively. It is also worth noting that the experimental values saturate similarly as the theoretical ones. The trend of the increasing difference between octahedral- and tetrahedral-site self-trapping energies originates from the decrease of the zero-point energy and motional amplitude. It is therefore a universal property, independent of the details of the hydrogen potential. It is thus not surprising that Sugimoto and Fukai $^{14}$ also reproduce this trend by constructing the hydrogen potential from a Born-Mayer-type pair potential, which shows a remarkably stronger short-range repulsion than the effective-medium potential. The measured ${ }^{31}$ temperature-independent prefactor of the diffusion constant in bec metals does not show the $1 / \sqrt{M}$ dependence on the isotope mass $M$, as the classical oscillator model predicts. This is an indication that the potential for hydrogen is indeed strongly anharmonic.

\section{Excited states of hydrogen in bcc metals}

We have analyzed the excited vibration states for hydrogen isotopes self-trapped in the tetrahedral site of the bcc metal $\mathrm{Nb}$. In the calculations the host-ion displacements of the ground state are used, i.e., the adiabatic (Born-Oppenheimer) approximation is used. The adiabat- 


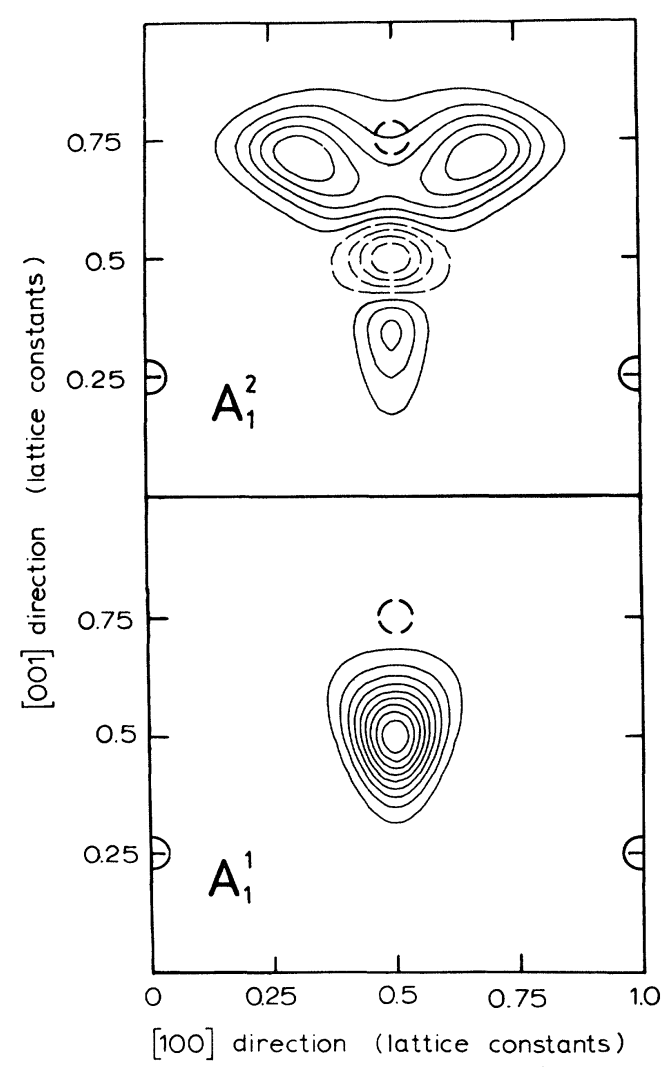

FIG. 6. Two lowest-energy $A_{1}$ wave functions for hydrogen self-trapped in the tetrahedral site in $\mathrm{Nb} . A_{1}^{1}$ is the ground state. The positions of the nearest $\mathrm{Nb}$ nuclei are denoted by semicircles (nucleus on the figure plane) or by dashed circles (nucleus above or below the figure plane). Negative values are shown by dashed contours. The contour spacing is one-tenth of the difference between the maximum and minimum values.

ic approximation implies that the difference between the excited- and ground-state energies should correspond to the neutron energy loss in neutron-scattering experiments. The tetrahedral site in the bcc structure has the symmetry of the $D_{2 d}$ point group. ${ }^{32}$ The excited states belonging to the representations $A_{1}, A_{2}, B_{1}, B_{2}$, and $E$ were searched for by imposing the proper boundary conditions, and with the use of an orthogonalization process. These techniques are described in Appendix A. The $E$ states are doubly degenerate, while the other states are nondegenerate. The $A_{1}$ states are the totally symmetric states, and $A_{1}^{1}$ is the ground state. In the case of the $\mathrm{Nb}$ host no self-trapped $A_{2}$ and $B_{1}$ states for $\mathrm{H}$ exist, i.e., the excitation energies exceed the self-trapping energy. Figures 6,7 , and 8 show the hydrogen wave functions for the two lowest $A_{1}, B_{2}$, and $E$ states, respectively. Qualitatively, $B_{2}^{1}$ and $E^{2}$ of the excited states are localized near the (trap) tetrahedral site. Other excited states are strongly shifted towards the four tetrahedral sites adjacent to the original one. The energies of the lowest $A_{1}, B_{2}$, and $E$ states for the three hydrogen isotopes are collected in Table IV.

The comparison of the calculated excitation energies with the inelastic-neutron-scattering data ${ }^{33}$ gives additional information about the potential experienced by the

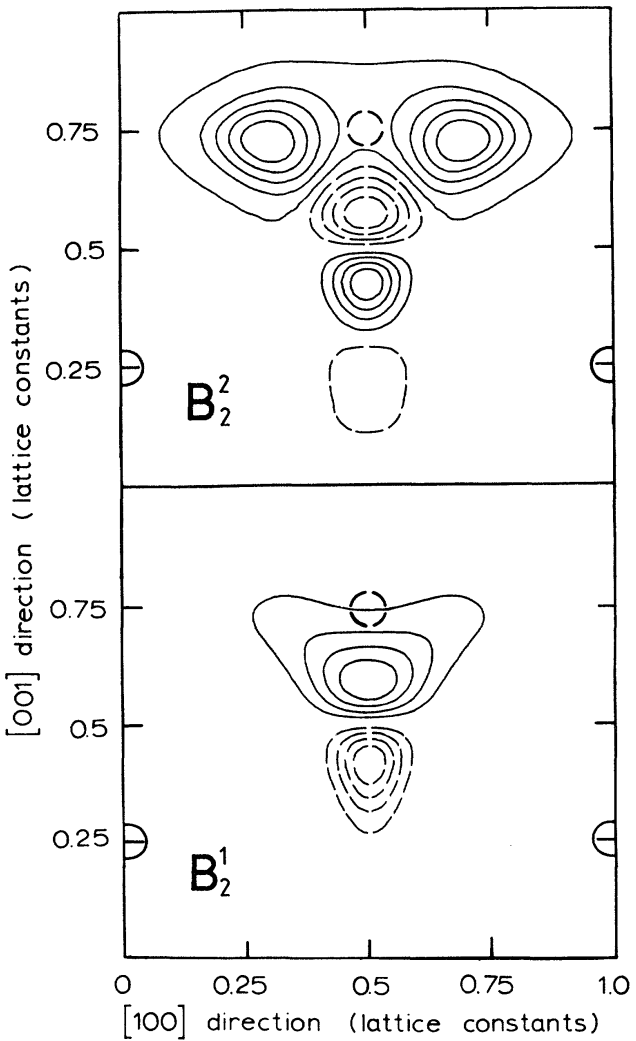

FIG. 7. Two lowest-energy $B_{2}$ wave functions for hydrogen self-trapped in the tetrahedral site in $\mathrm{Nb}$. For more details, see the caption of Fig. 6.

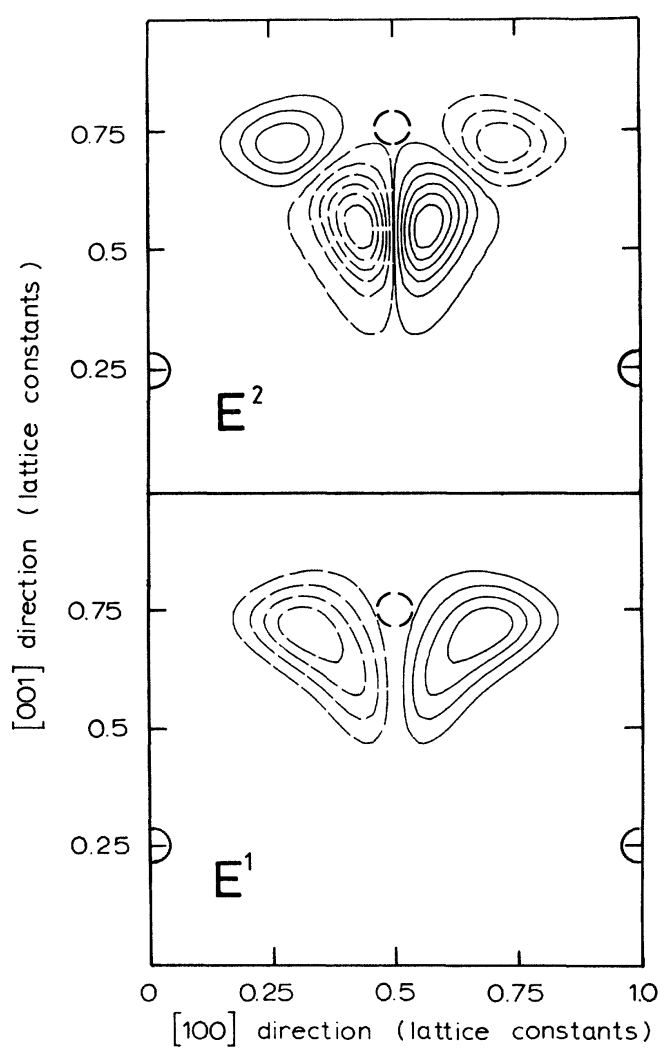

FIG. 8. Two lowest-energy $E$ wave functions for hydrogen self-trapped in the tetrahedral site in Nb. For more details, see the caption of Fig. 6. 
TABLE IV. Energies of the lowest excited vibrational states for hydrogen isotopes self-trapped in the tetrahedral site in $\mathrm{Nb}$. For further details, see Appendix A.

\begin{tabular}{crrr}
\hline \hline & \multicolumn{3}{c}{ Excitation energy $(\mathrm{meV})$} \\
Excited state & H & D & T \\
\hline$A_{1}^{2}$ & 72 & 69 & 66 \\
$A_{1}^{3}$ & 131 & 101 & 86 \\
$B_{2}^{1}$ & 48 & 37 & 31 \\
$B_{2}^{2}$ & 80 & 74 & 72 \\
$B_{2}^{3}$ & 135 & 109 & 95 \\
$\boldsymbol{E}^{1}$ & 73 & 63 & 53 \\
$\boldsymbol{E}^{2}$ & 95 & 75 & 73 \\
$\boldsymbol{E}^{3}$ & 149 & 101 & 88 \\
\hline \hline
\end{tabular}

self-trapped hydrogen. However, the interpretation of the experimental results is not very clear due to the role of hydrogen-hydrogen interactions in the experimentally studied metal-hydrogen alloys with the necessary relatively high hydrogen concentrations. Both in the energy-gain and energy-loss spectra, two peaks at $\sim 120$ and $\sim 170$ $\mathrm{meV}$ are visible, with an intensity ratio of $1: 2$ for all vanadium group hydrides. ${ }^{33}$ These results are conventionally interpreted by connecting the $170-\mathrm{meV}$ peak to a doubly degenerate state and the $120-\mathrm{meV}$ peak to a nondegenerate state. Compared with these experimental values the excitation energies of our calculations in Table IV are generally too low. The upper limit of the calculated excitation energies is the self-trapping energy, $151 \mathrm{meV}$. Higher excitations are possible in our model if the final state is an excited band state, as in the model discussed by Casella. ${ }^{16}$ One obvious reason for the smallness of the theoretical values is the softness of the effective-medium potential in the ion-core region, as discussed above. The transition probability to the $B_{2}^{1}$ and $E^{2}$ states is expected to be much larger than to the other excited states, which have a small overlap with the ground state leading to a small transition matrix element. ${ }^{15}$ Thus, as also discussed by Sugimoto and Fukai, ${ }^{15}$ it is tempting to assign the nondegenerate $B_{2}^{1}$ and the doubly degenerate $E^{2}$ states to the experimental 120- and 170-meV peaks, respectively. This conclusion is supported by the fact that the energy-loss and energy-gain spectra have peaks at the same energies. Thus the lattice deformation should be similar in the ground state and excited state. This, in turn, requires the hydrogen distributions of both states to be nearly equal, which is true only for $B_{2}^{1}$ and $E^{2}$ states. The experimental spectra contain, in addition, a rather high background. The background could be due to the less probable transitions to the $A_{1}^{2}, E^{1}$, and $B_{2}^{2}$ states. It is interesting to note from the calculations that these states resemble each other in energy.

The isotope dependence of the excitation energies gives further evidence for the interpretation that the experimental 120- and 170-meV peaks correspond to the $B_{2}^{1}$ and $E^{2}$ states. Namely, the isotope dependencies of these excitations are nearer to the experimentally observed harmonic-oscillator $1 / \sqrt{M}$ behavior than those of the
$A_{1}^{2}, E^{1}$, and $B_{2}^{2}$ states. The breakdown of the $1 / \sqrt{M}$ dependence for the latter is evident from Figs. 6-8, which show that the corresponding wave functions are very far from the solutions to a harmonic oscillator. It is interesting that, in the case of the $E^{2}$ state, the orthogonality repulsion due to the $E^{1}$ state makes the "effective" potential rather harmonic if we compare the energies for $\mathbf{H}$ and $\mathrm{D}$. It is, of course, difficult to draw firm conclusions because the effective-medium theory is not able to absolutely reproduce the experimental energy scale. However, the qualitative features of the wave functions as well as the trends in the excitation energies are not very sensitive to the potential construction. Thus, Sugimoto and Fukai ${ }^{15}$ found qualitatively the same wave functions using a pair potential fitted to the experiments. One fitting requirement they used was that the energy of the $E^{2}$ state was to be $170 \mathrm{meV}$. With this potential they were able to reproduce the $120-\mathrm{meV}$ peak as the $B_{2}^{1}$ state, and also found the $1 / \sqrt{M}$ dependence for the $E^{2}$ and $B_{2}^{1}$ states.

\section{C. fcc metals}

Figure 9 shows the hydrogen wave functions along the three principal directions in $\mathrm{Cu}$ when hydrogen is selftrapped in the octahedral site. The wave function is nearly isotropic; it is only slightly enhanced in the $\langle 111\rangle$ directions when compared with the $\langle 100\rangle$ and $\langle 110\rangle$ directions. The quantative results are given in Table $V$. The self-trapped state for the tetrahedral site was searched for by requiring that the wave function is localized in a cube with its center at the tetrahedral site and its corners

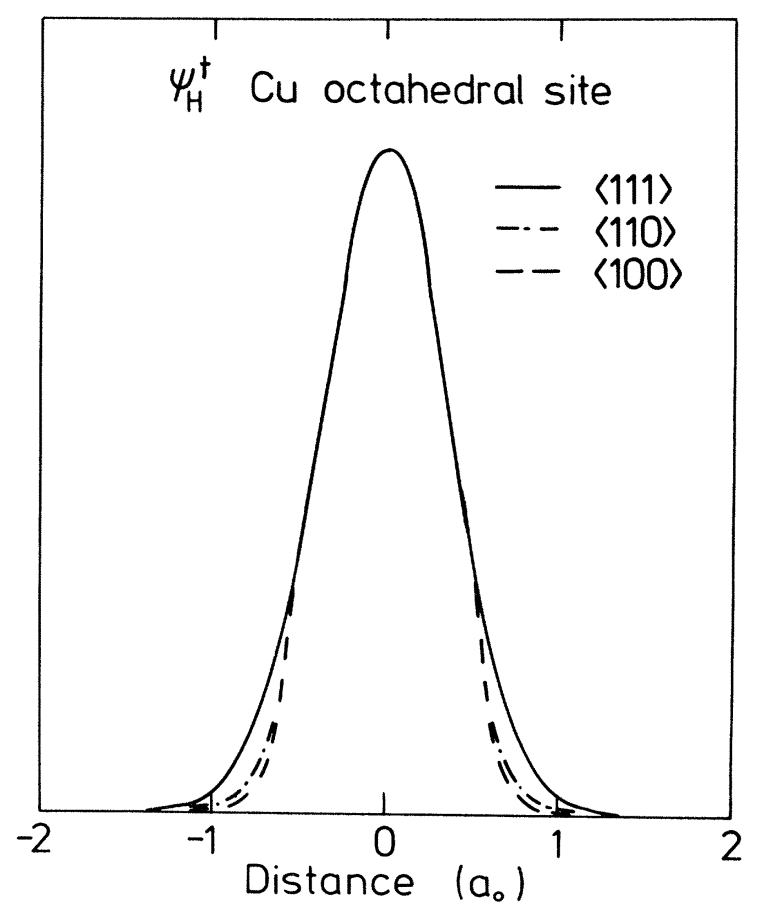

FIG. 9. Hydrogen wave function self-trapped in the octahedral site in $\mathrm{Cu}$. The wave function is drawn along the $\langle 100\rangle$ (dashed line), $\langle 110\rangle$ (dashed-dotted line), and $\langle 111\rangle$ (solid line) directions. The distance is measured from the octahedral point. 
TABLE V. Calculated properties of hydrogen self-trapped in octahedral $(O)$ and tetrahedral $(T)$ sites in fcc metals. For further details, see the caption of Table II.

\begin{tabular}{|c|c|c|c|c|c|c|c|c|}
\hline \multirow[b]{2}{*}{ Metal } & \multirow[b]{2}{*}{ Site } & \multirow{2}{*}{$\begin{array}{c}\text { Green } \\
\text { function }\end{array}$} & \multirow{2}{*}{$\begin{array}{l}\Delta E_{\alpha} \\
(\mathrm{eV})\end{array}$} & \multirow{2}{*}{$\begin{array}{c}E_{L} \\
(\mathrm{eV})\end{array}$} & \multirow{2}{*}{$\begin{array}{c}E_{0} \\
(\mathrm{eV})\end{array}$} & \multirow{2}{*}{$\begin{array}{l}E_{\mathrm{ST}} \\
(\mathrm{eV})\end{array}$} & \multicolumn{2}{|c|}{ Displacements $(\%)$} \\
\hline & & & & & & & First neighbors & Second neighbors \\
\hline \multirow[t]{2}{*}{$\mathbf{A l}$} & $O$ & $E$ & -0.102 & 0.046 & 0.076 & -0.056 & 3.3 & 0 \\
\hline & $T$ & $E$ & -0.008 & 0.099 & 0.079 & +0.091 & 6.8 & 0 \\
\hline \multirow[t]{2}{*}{$\mathrm{Ni}$} & $O$ & $E$ & -0.197 & 0.083 & 0.172 & -0.114 & 2.5 & 0.01 \\
\hline & $T$ & $\bar{E}$ & +0.097 & 0.229 & 0.138 & +0.326 & 5.9 & -0.02 \\
\hline \multirow[t]{4}{*}{$\mathrm{Cu}$} & $O$ & $L$ & -0.146 & 0.066 & 0.145 & -0.080 & 2.5 & 0.02 \\
\hline & $T$ & $L$ & +0.087 & 0.194 & 0.149 & +0.280 & 6.2 & -0.02 \\
\hline & $O$ & $E$ & -0.131 & 0.059 & 0.147 & -0.072 & 2.2 & -0.14 \\
\hline & $T$ & $E$ & +0.125 & 0.168 & 0.127 & +0.293 & 5.3 & -0.14 \\
\hline $\mathbf{P d}$ & $O$ & $E$ & -0.087 & 0.040 & 0.152 & -0.047 & 1.6 & -0.21 \\
\hline
\end{tabular}

at the nearest atoms or the octahedral points. With these boundary conditions the self-trapping to the tetrahedral interstice does not occur for any of the fcc metals studied, as indicated by the positive self-trapping energies in Table V. However, $\mathrm{Al}$ is, due to its relatively large lattice constant, a borderline case with a nearly vanishing selftrapping energy. Small changes in the boundary conditions and in the potential might also allow self-trapping in the tetrahedral site in Al. The self-trapping energy gain in the octahedral site is also relatively low in all cases. Surely, this is partly due to the softness of the effectivemedium potential, but we again emphasize that we will draw the conclusions from the general trends and differences, which are independent on the details of the potential.

Thus, lattice relaxation is small, consistent with the low trapping energy, in the vicinity of the hydrogen selftrapped in the octahedral site. The first-nearest neighbors relax outward by $2-3 \%$, and the displacements for the second-nearest neighbors almost vanish. If self-trapping were possible in the tetrahedral site, then the associated relaxation of the first-nearest neighbors would be larger, about $6 \%$ according to the Table V. The replacement of the elastic Green functions by the discrete-lattice Green functions does not alter the trends in the energy terms or the magnitudes of relaxation as seen in the case of $\mathrm{Cu}$.

The comparison between the properties of different hydrogen isotopes and $\mu^{+}$is presented in Table VI. The host metal is $\mathrm{Cu}$ and the energy terms are shown for the band state and the self-trapped octahedral state. The self-trapping energy decreases as the mass of the isotope increases. This is because the zero-point energy decreases more rapidly as a function of the mass in the band state than in the localized state. The rise of the bottom of the potential with the increase of the mass is compensated for by the decrease of lattice-deformation energy.

There are several published calculations concerning the trapping site of hydrogen in $\mathrm{Al}$ and the associated lattice relaxation. ${ }^{34-38}$ In these calculations, both octahedral and tetrahedral locations have been predicted for hydrogen. Theories where the electron density is calculated selfconsistently in the jellium model, and also where the discrete lattice is treated by perturbation theory, predict that either the octahedral ${ }^{34,35}$ or the tetrahedral ${ }^{36}$ site is the stable one. The octahedral site is the result in the spherical-solid model, ${ }^{35,37}$ where the spherical average of the host-ion pseudopotentials is included in the calculation of the electron density. In addition, Perrot and Rasolt ${ }^{37}$ allow the ions surrounding the hydrogen to relax radially. Solt et al. ${ }^{38}$ used the jellium model with lattice re-

TABLE VI. Isotope effects for hydrogenic impurities in Cu. Elastic Green function is used in the calculations. For further details, see the caption of Table III.

\begin{tabular}{lcrrr}
\hline \hline & & $\mu$ & $\mathrm{H}$ & \multicolumn{1}{c}{$\mathrm{D}$} \\
\hline Extended state & $E_{\alpha}$ & -1.443 & -1.849 & -1.907 \\
& $E_{0}$ & 0.581 & 0.175 & 0.117 \\
Self-trapped state & $E_{\alpha}$ & -1.658 & & -2.023 \\
& $E_{0}$ & 0.496 & -1.980 & 0.101 \\
& $E_{L}$ & 0.094 & 0.147 & 0.052 \\
& $E_{\mathrm{ST}}$ & -0.121 & 0.059 & -0.064 \\
Displacements (\%) & & & -0.072 & \\
First neighbors & & & & 2.1 \\
Second neighbors & & -0.18 & 2.2 & -0.13 \\
\hline \hline
\end{tabular}


laxation and found the octahedral and tetrahedral states to have nearly equal energies, the tetrahedral site being slightly more favorable. For $\mu^{+}$it is possible to determine also experimentally the stable location by muonspin-rotation techniques and it is found that muons are trapped by octahedral interstices at low temperatures in $\mathrm{Al}$ (Ref. 39) and $\mathrm{Cu}$ (Ref. 40).

The model by Perrot and Rasolt ${ }^{37}$ gives a relaxation of $2.2 \%$ for the first-nearest neighbors in the vicinity of hydrogen or $\mu^{+}$self-trapped in the octahedral site in Al. Solt et al. ${ }^{38}$ calculated the relaxation to be $2.5 \%$. These values are less than our estimate of $3.3 \%$ for the relaxation caused by $\mu^{+}$. From the muon-spin-rotation experiments the relaxation of the first-nearest neighbors is estimated to be relatively large, about 5\%, both in Al (Ref. 41) and in $\mathrm{Cu}$ (Ref. 40).

\section{Diffusion model in fcc metals}

Our results suggest a diffusion model for hydrogen in fcc metals that is totally different from the one described for bec metals. First we note that in the high-temperature region the diffusion activation energy in fcc metals is typically $0.2-0.4 \mathrm{eV},{ }^{31}$ which is much more than the calculated self-trapping energies in the octahedral sites. On the other hand, according to the calculations, self-trapping in the tetrahedral site is unprobable, and thus hydrogen would not be localized at the tetrahedral site during the activation process, but its wave function should be spread over several interstices. This also means that the activation energy is proportional to (or behaves in the same way as) the self-trapping energy. The decrease of calculated self-trapping energy as the mass of the isotope increases is thus consistent with the observed "reverse" isotope effect, ${ }^{31}$ i.e., in $\mathrm{Pd}, \mathrm{Ni}$, and $\mathrm{Cu}$ the activation energy for $\mathrm{D}$ is less than that for $H$. According to the experiments, the temperature-independent prefactor of the diffusion constant for $\mathrm{H}, \mathrm{D}$, and $\mathrm{T}$ behaves as the harmonic-oscillator model predicts, i.e., the ratio of the factors is $1: \sqrt{2}: \sqrt{3} \cdot{ }^{31}$ Thus the energy level of the hydrogen isotope should be located near the bottom of the self-trapping potential well. On the other hand, the prefactor for $\mu^{+}$is not obtained by using the mass extrapolation; ${ }^{42}$ this means that the zeropoint energy for $\mu^{+}$exceeds the harmonic region of the potential.

\section{HELIUM IN METALS}

The quantum nature of helium atoms is profoundly manifested in the properties of helium fluids at low temperatures. Here we investigate the importance of zeropoint effects for ${ }^{4} \mathrm{He}$ atoms injected in metals. The electronic aspects of helium injected in metals have been discussed by many workers, e.g., Inglesfield and Pendry, ${ }^{43}$ Whitmore, ${ }^{44}$ and Benedek. ${ }^{45}$ The crucial feature is naturally the strong orthogonality repulsion between the conduction electrons and the occupied $\mathrm{He}$ core.

The covalent-bonding term (22) can be neglected altogether, except for the consideration of the weak physisorption well outside surfaces, in the interaction energy of helium with metals. ${ }^{5,46}$ The bound $1 s$ level in $\mathrm{He}$ is well below the metal conduction band, and the hybridization is weak. The immersion energy $\Delta E_{\text {hom }}$ is a linear function of the electron density $n_{0}$ (Ref. 24) ( $n_{0}$ in units
TABLE VII. Dependence of the helium effective-medium potential on the cutoff radius $R_{a}$. Hydrogen is embedded in the unrelaxed octahedral site in $\mathrm{Cu} . \quad \bar{n}_{0}$ and $\alpha_{a}$ are defined in Eqs. (19) and (21), respectively. $V$ is calculated via Eq. (28).

\begin{tabular}{lccc}
\hline \hline $\begin{array}{l}R_{a} \\
\left(a_{0}\right)\end{array}$ & $\begin{array}{c}\bar{n}_{0} \\
\left(a_{0}^{-3}\right)\end{array}$ & $\begin{array}{c}\alpha_{a} \\
\left(\mathrm{eV} a_{0}^{3}\right)\end{array}$ & $\begin{array}{c}V \\
(\mathrm{eV})\end{array}$ \\
\hline 2 & 0.0203 & 98.7 & 3.57 \\
2.5 & 0.0218 & 121 & 3.35 \\
3 & 0.0236 & 134 & 3.34 \\
\hline \hline
\end{tabular}

$$
\begin{aligned}
& \text { of } a_{0}^{-3} \text { and } \Delta H_{\text {hom }} \text { in eV), } \\
& \qquad \Delta H_{\text {hom }}\left(n_{0}\right)=\left\{\begin{array}{l}
305 n_{0}, n_{0}<0.01 a_{0}^{-3} \\
275 n_{0}+0.3, n_{0} \geq 0.01 a_{0}^{-3}
\end{array}\right.
\end{aligned}
$$

The linear dependence of the helium-metal repulsive interaction on the electron density has also emerged from Hartree-Fock - type calculations for He-metal surface interactions. ${ }^{47}$ The first-order correction involves the coefficient $\alpha_{a}$ [Eq. (14)], which depends on the quasiatom electrostatic potential $\phi_{a}(\overrightarrow{\mathrm{r}})$. To first order, the interaction energy remains linearly proportional to $n_{0}$ and repulsive,

$$
V(\overrightarrow{\mathrm{r}})=\Delta E_{\text {hom }}\left[\bar{n}_{0}(\overrightarrow{\mathrm{r}})\right]-126 \bar{n}_{0}(\overrightarrow{\mathrm{r}}) \mathrm{eV} .
$$

In the case of $\mathrm{He}$, there is only a weak dependence of $\phi_{a}(\vec{r})$ on the density, which reflects the nearly inert and compact nature of the quasiatom electronic structure. The dependence on the cutoff radius is also small due to the small atomic radius. This is demonstrated in Table VII, which lists the relevant numbers for a stationary $\mathrm{He}$ atom embedded in the octahedral interstice in fcc $\mathrm{Cu}$. The electron density of both the host and the impurity is approximated in terms of atomic densities. Table VII shows that the total interaction potential $V$ is well saturated when the cutoff radius $R_{a} \approx 2.5 a_{0}$. In the calculations we have used this cutoff radius and atomic electron density for He.

We have employed our approach to describe the self-

TABLE VIII. Self-trapping characteristics for He in octahedral $(O)$ and tetrahedral $(T)$ sites in Al. Elastic Green function is used in the calculations. All energies are in $\mathrm{eV}$. For further details, see the caption of Table II.

\begin{tabular}{llcr}
\hline \hline Extended state & $E_{\alpha}$ & 3.255 & \\
& $E_{0}$ & 0.092 & \\
& & & $T$ \\
\hline Self-trapped state & $E_{\alpha}$ & 2.189 & 2.210 \\
& $E_{0}$ & 0.053 & 0.069 \\
& $E_{L}$ & 0.403 & 0.612 \\
& $E_{\mathrm{ST}}$ & -0.663 & -0.434
\end{tabular}

Displacements (\%)

First neighbors 9.8

Second neighbors

0.07

17.2

0 
TABLE IX. Self-trapping characteristics for He in octahedral $(O)$ and tetrahedral $(T)$ sites in $\mathrm{Nb}$. Green function of the discrete lattice theory is used (Ref. 19). All energies are in $\mathrm{eV}$. For further details, see the caption of Table II.

\begin{tabular}{lccr}
\hline \hline Extended state & $E_{\alpha}$ & 4.555 & \\
& $E_{0}$ & 0.123 & \\
& & & $T$ \\
\hline Self-trapped state & $E_{\alpha}$ & 3.043 & 3.101 \\
& $E_{0}$ & 0.082 & 0.093 \\
& $E_{L}$ & 0.606 & 0.551 \\
& $E_{\mathrm{ST}}$ & -0.906 & -0.903
\end{tabular}

Displacements $(\%)$

\begin{tabular}{lrr} 
First neighbors & 16.5 & 9.2 \\
Second neighbors & 1.0 & -0.6 \\
\hline \hline
\end{tabular}

trapping of helium in fcc $\mathrm{Al}$ and in bcc $\mathrm{Nb}$. The results are collected in Tables VIII and IX. For the extended (unrelaxed) state, the heat of solution is very high and compares well with earlier estimates. The zero-point kinetic component is of the order of $0.1 \mathrm{eV}$ and of minor importance. Allowing self-trapping in fcc Al, the octahedral site is clearly more favorable than the tetrahedral site. In bcc $\mathrm{Nb}$, octahedral and tetrahedral sites are, also for $\mathrm{He}$, nearly degenerate in energy. The relaxation caused by $\mathrm{He}$ is much larger than in the case of the hydrogenic isotopes. Furthermore, the self-trapping energy is now much larger.

It is quite clear that helium will favor any open-volume defects present in the metal, and will trap extremely strongly to, e.g., vacancies and their aggregates. In fact, the self-trapping energy gain is similar in size to the vacancy-formation energy, which points to the possibility of helium actually driving the vacancy generation. The vacancy generation has been demonstrated previously by Wilson et al. ${ }^{48}$ who studied the energetics of helium clustering in $\mathrm{Ni}$. In their calculations, movable $\mathrm{Ni}$ and $\mathrm{He}$ atoms interact via pair potentials, and they found that 5-6 He atoms spontaneously produce a vacancy-selfinterstitial pair during the clustering process. The trapping energy of the helium to a vacancy, once created, is, of course, higher than the self-trapping energy in an interstitial site. Manninen et al. ${ }^{9}$ have investigated the trapping of one or several helium atoms into pre-existing monovacancies in transition metals. If one includes the zero-point and lattice-relaxation energies estimated here in their calculations, the results for the trapping energies are shifted to closer agreement with the experimental values based on thermal-desorption measurements. ${ }^{49}$ The relaxation effects are expected to be minor for substitutional helium.

In the self-trapped interstitial state the total embedding energy is high and positive. In the case of $\mathrm{Al}$ it corresponds to a swelling pressure of the order

$$
P \approx 3 E_{\alpha} / 4 \pi R_{a}^{3} \approx 10^{11} \mathrm{~Pa},
$$

which is comparable to the elastic yield limit of metals.
Thus it is possible for helium-filled bubbles to form and grow in the metal matrix. An interesting question is the equilibrium helium density in a void of given size. ${ }^{50}$ With the techniques presented here, the equilibrium energetics (equation of state) should be fairly easy to calculate, but the complete analysis is hampered by the lack of understanding of the details of the bubble-growth mechanisms.

\section{CONCLUDING REMARKS}

The quasiatom concept (effective-medium theory) combined with a wave-mechanical treatment of the impurity degree freedom and a self-consistent calculation of the lattice distortions form a powerful approach to detailed understanding of the properties of light interstitials in metals. Results of calculations involving all these aspects have been presented here. The qualitative trends discovered here provide a coherent picture of many observed static features. Two important general conclusions emerge: (i) for hydrogen (and $\mu^{+}$) the quantum nature is crucial, and (ii) contrary to the case of $\mathrm{He}$, no description based on pairwise potentials is truly adequate for hydrogen. However, in order to obtain numerically accurate $a b$ initio-type results for a wide class of systems, further improvements seem necessary in the weakest link of the method, the potential-energy construction. Unfortunately, at least with the present state of knowledge, it is not possible to obtain corrections to the effective-medium theory in a systematic fashion. One then has to either abandon striving for quantitative accuracy and examine qualitative trends, or to resort to parametrization. It is obvious that both of these routes have much to contribute to our understanding of light impurities in metallic media.

\section{ACKNOWLEDGMENTS}

This research has been supported in part by the Academy of Finland. We are grateful to Matti Manninen and Jens Nørskov for numerous useful discussions.

\section{APPENDIX A: NUMERICAL SOLUTION OF THE THREE-DIMENSIONAL SCHRÖDINGER EQUATION}

The solution of the wave function for the full threedimensional potential is accomplished in this work by a numerical relaxation technique first presented by Kimball and Shortley. ${ }^{51}$ For localized states the wave function is required to vanish on the surface of a sufficiently large polyhedron enclosing the trap site. Propagating band states are obtained by constructing the polyhedron with the use of high-symmetry (e.g., reflection) planes of the lattice, and imposing proper boundary conditions on them. The boundary conditions depend on the Bloch wave vector and symmetry. In the numerical solution, a mesh of points is embedded in the smallest (irreducible) wedge of the polyhedron allowed by the symmetry of the lattice and the eigenfunction in question.

If the mesh of the points is chosen to build up a simple cubic lattice, the wave function $\psi$ is solved by the iteration process (in a.u.), 


$$
\psi_{i j k}^{n+1}=\frac{\psi_{i+1, j, k}^{n}+\psi_{i-1, j, k}^{n}+\psi_{i, j+1, k}^{n}+\psi_{i, j-1, k}^{n}+\psi_{i, j, k+1}^{n}+\psi_{i, j, k-1}^{n}}{6+2 m h^{2}\left(V_{i j k}-E^{n}\right)},
$$

where the indices $i, j$, and $k$ enumerate the mesh points, the superscripts give the order of the iteration, $h$ is the spacing of the mesh, and $m$ is the mass of the quantum particle. Equation (A1) is derived variationally from the Schrödinger equation by substituting a simple difference scheme for the Laplace operator. The energy eigenvalue $E^{n}$ in (A1) is calculated from the difference formula

$$
E^{n}=\left[\sum_{i, j, k}-\frac{\psi_{i j k}^{n}}{2 m h^{2}}\left(\psi_{i+1, j, k}^{n}+\psi_{i-1, j, k}^{n}+\psi_{i, j+1, k}^{n}+\psi_{i, j-1, k}^{n}+\psi_{i, j, k+1}^{n}+\psi_{i, j, k-1}^{n}-6 \psi_{i j k}^{n}\right)\right] /\left[\sum_{i, j, k}\left(\psi_{i j k}^{n}\right)^{2}\right) .
$$

The iterations are started by first guessing some form for the wave function and calculating the corresponding energy estimate. Then a new wave function and energy value are determined from Eqs. (A1) and (A2). In a stable iteration process the energy eigenvalue decreases monotonically between iterations. We have continued the iterations until the energy eigenvalue is stationary within the computer accuracy.

Higher-energy eigenstates are obtained most easily by orthogonalizing the wave function after each iteration (A1) against the lower-energy states stored in the computer memory. The adequate numerical procedure is ${ }^{51}$

$$
\phi_{i j k}^{n, \alpha+1}=\psi_{i j k}^{n, \alpha+1}-\sum_{\beta=1}^{\alpha} M^{\beta} \phi_{i j k}^{\beta},
$$

where

$$
M^{\beta}=\left(\sum_{i, j, k} \phi_{i j k}^{\beta} \psi_{i j k}^{\alpha+1}\right) /\left(\sum_{i, j, k}\left(\phi_{i j k}^{\beta}\right)^{2}\right) .
$$

The $\beta$ summation runs over the $\alpha$ lowest-energy states. $\psi$ refers to the unorthogonalized state and the $\phi$ 's refer to the orthogonalized ones.

The iteration scheme described is stable if the mesh is fine enough ${ }^{51}$ and the stability does not depend strongly on the starting wave function. The point density in the mesh is limited by the computer capacity: In one spatial direction we normally use $20-30$ points, which is suffi-

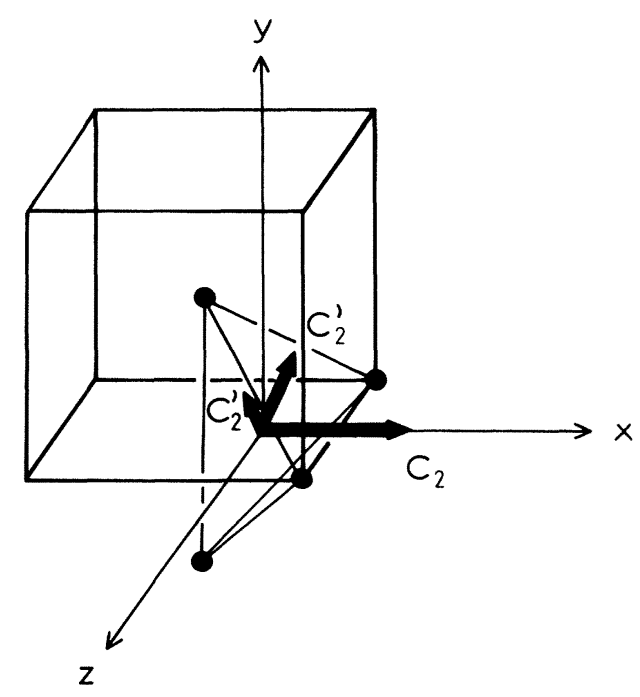

FIG. 10. Tetrahedral site in the bcc structure. The $C_{2}$ and $C_{2}^{\prime}$ rotation axes are shown. The $C_{2}$ axis also coincides with the axis of the improper rotation $S_{4}$. The $\sigma_{d}$ reflection planes are $y=0$ and $z=0$. cient for the required accuracy. Note also that the increase of the point density increases the number of iterations needed very rapidly.

In practice, all the reduction allowed by the symmetry has to be used in order to obtain accurate and stable solutions, both for the ground state and the excited states. Group theory facilitates the classification of the different states and gives proper boundary conditions. We illustrate these points by using the hydrogen energy levels in tetrahedral site of the bcc lattice as examples.

The tetrahedral site in bcc structure has the symmetry of $D_{2 d}$ point group ${ }^{32}$ containing the operations $E, C_{2}, C_{2}^{\prime}$, $S_{4}$, and $\sigma_{d}$ (see Fig. 10). There are five different representations, $A_{1}, A_{2}, B_{1}, B_{2}$, and $E$, and the character table for these representations is presented as Table $X$. The boundary conditions for states belonging to the onedimensional representations are easily deduced from the character table. The lowest-energy and excited states belonging to the same particular representation can then be calculated by the orthogonalization process. $A_{1}$ states are totally symmetric, i.e., they are invariant under all group operations. Thus, requiring the first normal derivative to vanish on the reflection planes $y=0$ and $z=0$ (Fig. 10), and utilizing the improper rotation symmetry over $x=0$ plane, the wave functions for different $A_{1}$ states are solved in one-eighth of the total polyhedron enclosing the wave function. According to the character table the wave functions belonging to the $A_{2}$ representation change sign in the $\sigma_{d}$ reflections and $C_{2}^{\prime}$ rotations. Thus the $A_{2}$ wave functions have $x=0, y=0$, and $z=0$ as node planes. Similarly, it is seen that $B_{1}$ wave functions vanish on the $y=0$ and $z=0$ planes, and $B_{2}$ wave functions vanish only on the $x=0$ plane. Solving in one-eighth of the polyhedron is also sufficient for the solution of the $A_{2}, B_{1}$, and $B_{2}$ states. The doubly degenerate states belonging to representation $E$ are obtained by requiring the wave function to vanish on the $z=0$ or $y=0$ plane. This boundary condition breaks the improper rotation symmetry, and, accordingly, the wave functions have to be solved in onefourth of the total polyhedron.

TABLE X. Character table for the $D_{2 d}$ point group.

\begin{tabular}{lrrrrr}
\hline \hline$D_{2 d}$ & $E$ & $C_{2}$ & $2 S_{4}$ & $2 C_{2}^{\prime}$ & $2 \sigma_{d}$ \\
\hline$A_{1}$ & 1 & 1 & 1 & 1 & 1 \\
$A_{2}$ & 1 & 1 & 1 & -1 & -1 \\
$B_{1}$ & 1 & 1 & -1 & 1 & -1 \\
$B_{2}$ & 1 & 1 & -1 & -1 & 1 \\
$E$ & 2 & -2 & 0 & 0 & 0 \\
\hline \hline
\end{tabular}




\section{APPENDIX B: REDUCED GREEN FUNCTIONS}

The restriction of the lattice relaxation in the two nearest-neighbor shells around the impurity and the use of the symmetry reduces the dimension of the Green-function matrix needed. Table XI gives the relations between the reduced Green functions and the Green functions $G_{i j}(\vec{R})$ defined in the text. Octahedral and tetrahedral interstices in bcc and fcc structure have been considered.

TABLE XI. Reduced Green functions $g$ for octahedral and tetrahedral sites in bcc and fcc metals. The notation $\Gamma_{\alpha}^{\beta}$ is used for the Green function $G_{i j}(\vec{R})$. The subscript $\alpha$ denotes the order of the nearest-neighbor shell and the superscript $\beta$ is the Voigt notation for indexes $i$ and $j$. The origin of the coordinate system is at the interstitial site. In this notation the displacements $\overrightarrow{\mathrm{u}}$ are deduced from the forces $\overrightarrow{\mathrm{f}}$ by using $\overrightarrow{\mathrm{u}}=g \overrightarrow{\mathrm{f}}$.

\begin{tabular}{|c|c|c|c|c|}
\hline $\begin{array}{l}\text { Lattice } \\
\text { site }\end{array}$ & $\begin{array}{l}\text { Neighboring atoms } \\
\text { (lattice constant }=1 \text { ) }\end{array}$ & Displacements & Forces & $\begin{array}{l}\text { Components of the reduced } \\
\text { Green functions }\end{array}$ \\
\hline bcc octahedral & $\begin{array}{l}( \pm 0.5,0,0) \\
(0, \pm 0.5, \pm 0.5)\end{array}$ & $\begin{array}{l}\left( \pm u_{1}, 0,0\right) \\
\left(0, \pm u_{2}, \pm u_{2}\right)\end{array}$ & $\begin{array}{l}\left( \pm f_{1}, 0,0\right) \\
\left(0, \pm f_{2}, \pm f_{2}\right)\end{array}$ & $\begin{array}{l}g_{11}=\Gamma_{0}^{1}-\Gamma_{2}^{1} \\
g_{12}=-8 \Gamma_{1}^{6} \\
g_{21}=\frac{1}{4} g_{12} \\
g_{22}=\Gamma_{0}^{1}+\Gamma_{2}^{2}-\Gamma_{2}^{1}-\Gamma_{3}^{1}-\Gamma_{3}^{6}\end{array}$ \\
\hline bcc tetrahedral & $\begin{array}{l}( \pm 0.5,0,-0.25) \\
(0, \pm 0.5,+0.25) \\
( \pm 0.5,0,0.75) \\
(0, \pm 0.5,-0.75)\end{array}$ & $\begin{array}{l}\left( \pm u_{1}, 0,-u_{2}\right) \\
\left( \pm u_{3}, 0, u_{4}\right)\end{array}$ & $\begin{array}{l}\left(f_{1}, 0,-f_{2}\right) \\
\left( \pm f_{3}, 0, f_{4}\right)\end{array}$ & $\begin{array}{l}g_{11}=\Gamma_{0}^{1}-\Gamma_{2}^{1}-2 \Gamma_{1}^{6} \\
g_{12}=-2 \Gamma_{1}^{6} \\
g_{13}=\Gamma_{2}^{2}-\Gamma_{3}^{1}-2 \Gamma_{1}^{6} \\
g_{14}=-2 \Gamma_{1}^{6}-\Gamma_{3}^{6} \\
g_{21}=g_{12} \\
g_{22}=\Gamma_{0}^{1}+\Gamma_{2}^{2}-2 \Gamma_{1}^{1} \\
g_{23}=2 \Gamma_{1}^{6}-\Gamma_{3}^{6} \\
g_{24}=2 \Gamma_{1}^{1}-\Gamma_{2}^{1}-\Gamma_{3}^{1} \\
g_{31}=g_{13} \\
g_{32}=g_{23} \\
g_{33}=\Gamma_{0}^{1}-\Gamma_{2}^{1}-2 \Gamma_{4}^{6} \\
g_{34}=-2 \Gamma_{4}^{6} \\
g_{41}=g_{14} \\
g_{42}=g_{24} \\
g_{43}=g_{34} \\
g_{44}=\Gamma_{0}^{1}+\Gamma_{2}^{2}-2 \Gamma_{4}^{1}\end{array}$ \\
\hline fcc octahedral & $\begin{array}{l}( \pm 0.5,0,0) \\
(0, \pm 0.5,0) \\
(0,0, \pm 0.5) \\
( \pm 0.5, \pm 0.5, \pm 0.5)\end{array}$ & $\begin{array}{l}\left( \pm u_{1}, 0,0\right) \\
\left( \pm u_{2}, \pm u_{2}, \pm u_{2}\right)\end{array}$ & $\left( \pm f_{2}, \pm f_{2}, \pm f_{2}\right)$ & $\begin{array}{l}g_{11}=\Gamma_{0}^{1}-4 \Gamma_{1}^{6}-\Gamma_{2}^{1} \\
g_{12}=4 \Gamma_{1}^{3}-4 \Gamma_{3}^{1}-8 \Gamma_{3}^{6} \\
g_{21}=g_{12} / 4 \\
g_{22}=\Gamma_{0}^{1}-\Gamma_{2}^{1}+2 \Gamma_{2}^{2}-2 \Gamma_{4}^{1}+\Gamma_{4}^{3}-2 \Gamma_{4}^{6}-\Gamma_{6}^{1}-2 \Gamma_{3}^{6}\end{array}$ \\
\hline fcc tetrahedral & $\begin{array}{l}(-0.25,-0.25,0.25) \\
(-0.25,0.25,-0.25) \\
(0.25,-0.25,0.25) \\
(0.25,0.25,0.25) \\
(-0.25,-0.25,-0.75) \\
(-0.25,-0.75,-0.25) \\
(0.25,0.25,-0.75) \\
(0.25,-0.75,0.25) \\
(-0.75,0.25,0.25)\end{array}$ & $\left(-u_{2},-u_{2},-u_{3}\right)$ & $\left(-f_{2},-f_{2},-f_{3}\right)$ & $\begin{array}{l}g_{11}=\Gamma_{0}^{1}-2 \Gamma_{1}^{1}-2 \Gamma_{1}^{6}+\Gamma_{1}^{3} \\
g_{12}=-2 \Gamma_{1}^{1}+2 \Gamma_{1}^{3}+2 \Gamma_{1}^{6}+2 \Gamma_{2}^{2}-2 \Gamma_{3}^{2}-2 \Gamma_{3}^{4}-2 \Gamma_{3}^{6} \\
g_{13}=-2 \Gamma_{1}^{6}+2 \Gamma_{1}^{1}-\Gamma_{2}^{1}-\Gamma_{3}^{1}-2 \Gamma_{3}^{6} \\
g_{21}=\frac{1}{2} g_{12} \\
g_{22}=\Gamma_{0}^{1}-\Gamma_{1}^{1}+\Gamma_{1}^{3}-2 \Gamma_{1}^{6}-2 \Gamma_{3}^{2}+\Gamma_{4}^{3}-\Gamma_{4}^{6}-\Gamma_{5}^{2}-\Gamma_{5}^{3} \\
g_{23}=\Gamma_{1}^{1}-\Gamma_{3}^{1}+\Gamma_{3}^{2}-\Gamma_{3}^{6}-\Gamma_{3}^{4}-\Gamma_{4}^{1}-\Gamma_{5}^{6} \\
g_{31}=g_{13} \\
g_{32}=2 g_{23} \\
g_{33}=\Gamma_{0}^{1}+\Gamma_{1}^{3}-2 \Gamma_{1}^{6}-4 \Gamma_{3}^{6}-2 \Gamma_{4}^{6}-2 \Gamma_{5}^{1}\end{array}$ \\
\hline
\end{tabular}

${ }^{1}$ For timely and recent reviews, see, e.g., Hydrogen in Metals, edited by P. Jena and C. B. Satterthwaite (Plenum, New York, 1983); Point Defects and Defect Interactions in Metals, edited by J. Takamura, M. Doyama, and M. Kiritani (NorthHolland, Amsterdam, 1982); E. Karlsson, Phys. Rep. 82, 271 (1982).

${ }^{2}$ See, e.g., P. H. Dederichs and R. Zeller, in Festkörperprobleme (Advances in Solid State Physics), edited by J. Treusch (Vieweg, Braunschweig, 1981), Vol. XXI; R. M. Nieminen, Phys. Scr. 25, 703 (1982); O. Gunnarsson, O. Jepsen, and O.
K. Andersen, Phys. Rev. B 27, 7144 (1983); O. Jepsen, R. M. Nieminen, and J. Madsen, Solid State Commun. 34, 575 (1980).

${ }^{3}$ M. J. Stott and E. Zaremba, Phys. Rev. B 22, 1564 (1980).

4J. K. Nørskov and N. D. Lang, Phys. Rev. B 21, 2316 (1980).

5J. K. Nørskov, Phys. Rev. B 26, 2875 (1982).

6J. K. Nørskov, Phys. Rev. Lett. 48, 1620 (1982).

${ }^{7}$ P. Nordlander, S. Holloway, and J. K. Nørskov, Surf. Sci. 136, 59 (1984).

${ }^{8}$ J. K. Nørskov, F. Besenbacher, J. Bottiger, B. B. Nielsen, and 
A. A. Pisarev, Phys. Rev. Lett. 149,1420 (1982).

${ }^{9}$ M. Manninen, J. K. Nørskov, and C. Umrigar, J. Phys. F $\underline{12}$, L7 (1982).

${ }^{10}$ N. Esbjerg and J. K. Nørskov, Phys. Rev. Lett. 45, 807 (1980); D. R. Hamann, ibid. 46, 1227 (1981); M. Manninen, J. K. Nørskov, and C. Umrigar, Surf. Sci. $\underline{119}$, L393 (1982).

${ }^{11}$ M. S. Daw and M. I. Baskes, Phys. Rev. Lett. 므, 1285 (1983).

${ }^{12}$ M. S. Daw, C. L. Bisson, and W. D. Wilson, Solid State Commun. 46, 735 (1983).

${ }^{13}$ See, e.g., Interatomic Potentials and Simulation of Lattice Defects, edited by P. C. Gehlen, J. R. Beeler, Jr., and R. I. Jaffee (Plenum, New York, 1972).

${ }^{14}$ H. Sugimoto and Y. Fukai, Phys. Rev. B 22, 670 (1980).

${ }^{15}$ H. Sugimoto and Y. Fukai, J. Phys. Soc. Jpn. 50, 3709 (1981).

${ }^{16}$ R. C. Casella, Phys. Rev. B 27, 5943 (1983).

${ }^{17}$ H. Wipf, A. Magerl, S. M. Shapiro, S. K. Satija, and W. Thomlinson, Phys. Rev. Lett. 46, 947 (1981).

${ }^{18} \mathrm{G}$. Leibfried and N. Breuer, in Point Defects in Metals I, Vol. 81 of Springer Tracts in Modern Physics (Springer, Heidelberg, 1978).

${ }^{19}$ V. K. Tewary, Adv. Phys. 22, 757 (1973).

${ }^{20}$ H. J. Kanzaki, J. Phys. Chem. Solids $\underline{2}$, 24 (1957).

${ }^{21}$ I. R. MacGillivray and C. A. Sholl, J. Phys. F $\underline{13}$, 23 (1983).

22P. H. Dederichs and G. Liebfried, Phys. Rev. 188, 1175 (1969).

${ }^{23}$ H. R. Schober, M. Mostoller, and P. H. Dederichs, Phys. Status Solidi B 64, 173 (1974).

${ }^{24}$ M. J. Puska, R. M. Nieminen, and M. Manninen, Phys. Rev. B $\underline{24}, 3037$ (1981).

${ }^{25}$ K. Mednick and L. Kleinman, Phys. Rev. B 22, 5678 (1980); C. S. Wang, A. Freeman, H. Kraukauer, and M. Posternak, ibid. 23, 1685 (1981); B. Chakraborty, R. W. Siegel, and W. E. Pickett, ibid. 24, 5445 (1981); R. Zeller and P. J. Braspenning, Solid State Commun. 42, 701 (1982).

26O. K. Andersen, Phys. Rev. B 12, 3060 (1975).

${ }^{27}$ J. K. Nørskov (private communication).

${ }^{28}$ H. Behr, H. M. Keppler, G. Steyrer, H. Metzger, and J. Peisl, J. Phys. F 13, L29 (1983).

${ }^{29}$ For reviews, see, e.g., S. T. Picraux, Nucl. Instrum. and Methods 182-183, 413 (1981); H.-D. Carstanjen, Phys. Status Solidi A 59, 11 (1980).

${ }^{30}$ D. Emin, Bull. Am. Phys. Soc. 28, 410 (1983); see also D. Emin, M. I. Baskes, and W. D. Wilson, Phys. Rev. Lett. 42, 791 (1979).
${ }^{31}$ Experimental results are collected in the review by J. Völk1 and G. Alefeld, in Hydrogen in Metals I, Vol. 28 of Topics in Applied Physics, edited by G. Alefeld and J. Völkl (Springer, Berlin, 1978), p. 321.

${ }^{32}$ Schönflies notation for the point groups and symmetry operations is used. See, e.g., M. Tinkham, Group Theory and Quantum Mechanics (McGraw-Hill, New York, 1964).

${ }^{33}$ Experimental results are collected in the review by $T$. Springer, in Hydrogen in Metals I, Ref. 31, p. 75.

${ }^{34}$ Z. D. Popovic, M. J. Stott, J. P. Carbotte, and G. R. Piercy, Phys. Rev. B $\underline{13}, 590$ (1976).

${ }^{35}$ M. Manninen and R. M. Nieminen, J. Phys. F 9, 1333 (1979).

${ }^{36}$ D. S. Larsen and J. K. Nørskov, J. Phys. F 9, 1975 (1979).

${ }^{37}$ F. Perrot and M. Rasolt, Phys. Rev. B 23, 6534 (1981).

${ }^{38}$ G. Solt, M. Manninen, and H. Beck, J. Phys. F 13,1379 (1983).

${ }^{39}$ K. W. Kehr, D. Richter, J.-M. Welter, O. Hartman, E. Karlsson, L. O. Norlin, T. O. Niinikoski, and A. Yaouanc, Phys. Rev. B 26, 567 (1982).

${ }^{40}$ M. Camani, F. N. Gygax, W. Ruegg, A. Schenck, and H. Schilling, Phys. Rev. Lett. 39, 836 (1977).

${ }^{41}$ D. Richter, in Nuclear and Electron Resonance Spectroscopies Applied to Materials Science, edited by N. Kaufman and G. K. Shenoy (North-Holland, New York, 1981).

${ }^{42}$ E. Karlsson, Phys. Rep. 82, 271 (1982).

${ }^{43}$ J. E. Inglesfield and J. B. Pendry, Philos. Mag. 34, 205 (1976).

${ }^{44}$ M. D. Whitmore, J. Phys. F $\underline{6}, 1259$ (1976).

${ }^{45}$ R. Benedek, J. Phys. F $\underline{8}, 807$ (1978).

${ }^{46}$ N. D. Lang and J. K. Nørskov, Phys. Rev. B 27, 4612 (1983).

${ }^{47}$ J. Harris and A. Liebsch, J. Phys. C 15, 2275 (1982); P. Nordlander and J. Harris (unpublished).

${ }^{48}$ W. D. Wilson, C. L. Bisson, and M. I. Baskes, Phys. Rev. B $\underline{24}$, 5616 (1981); M. I. Baskes and W. D. Wilson, ibid. 27, 2210 (1983).

${ }^{49}$ E. V. Kornelson, Radiat. Eff. 13, 227 (1972); E. V. Kornelson and D. E. Edwards, Jr., in Application of Ion Beams to Met$a l s$, edited by S. T. Picreaux, E. D. Eernisse, and F. L. Vook (Plenum, New York, 1974); Z. M. Caspers, A. van Veen, A. A. van Gorkum, A. van den Benkel, and C. B. van Baal, Status Solidi A 37, 371 (1976).

50See, e.g., R. Manzke, W. Jäger, H. Trinkaus, G. Crecelius, R. Zeller, and J. Fink, Solid State Commun. 44, 481 (1982); A. A. Lucas, J. P. Vigneron, S. E. Donnelly, and J. C. Rife, Phys. Rev. B 26, 2485 (1983).

${ }^{51}$ G. E. Kimball and G. H. Shortley, Phys. Rev. 45, 815 (1934). 